\title{
Documentation of Commonly Used Ethnoveterinary Medicines from Wild Plants of the High Mountains in Shimla District, Himachal Pradesh, India
}

\author{
Pramod Prakash ${ }^{1}$, Radha ${ }^{1, *}$, Manoj Kumar ${ }^{2, *(D)}$, Ashok Pundir ${ }^{3}$, Sunil Puri ${ }^{1}$, Suraj Prakash ${ }^{1}$ (D), \\ Neeraj Kumari ${ }^{1}{ }^{\mathbb{D}}$, Mamta Thakur ${ }^{1}$, Sonia Rathour ${ }^{1}$, Radhika Jamwal ${ }^{4}$, Salena Janjua ${ }^{5}$ De, Mansoor Ali ${ }^{5}$, \\ Sneh Punia Bangar ${ }^{6}{ }^{D}$, Chhatarpal Singh ${ }^{7}$, Deepak Chandran ${ }^{8}\left(\mathbb{D}\right.$, Sureshkumar Rajalingam ${ }^{9}$ (D), \\ Marisennayya Senapathy ${ }^{10}$, Sangram Dhumal ${ }^{11}$ (D), Surinder Singh ${ }^{12, *}$, Mahesh Kumar Samota ${ }^{13}$, \\ Rahul D. Damale ${ }^{14}$, Sushil Changan ${ }^{15}$, Suman Natta ${ }^{16} \mathbb{( D}$, Mohamd Alblihed ${ }^{17} \mathbb{( D}$, Attalla F. El-kott ${ }^{18,19}(\mathbb{D})$ \\ and Mohamed M. Abdel-Daim 20,21 (D)
}

Citation: Prakash, P.; Radha; Kumar, M.; Pundir, A.; Puri, S.; Prakash, S.; Kumari, N.; Thakur, M.; Rathour, S. Jamwal, R.; et al. Documentation of Commonly Used Ethnoveterinary Medicines from Wild Plants of the High Mountains in Shimla District, Himachal Pradesh, India. Horticulturae 2021, 7, 351. https:// doi.org/10.3390/horticulturae7100351

Academic Editor: Boris Duralija, Ksenija Karlović and Dario Kremer

Received: 30 August 2021

Accepted: 20 September 2021

Published: 1 October 2021

Publisher's Note: MDPI stays neutral with regard to jurisdictional claims in published maps and institutional affiliations.

Copyright: (C) 2021 by the authors Licensee MDPI, Basel, Switzerland. This article is an open access article distributed under the terms and conditions of the Creative Commons Attribution (CC BY) license (https:// creativecommons.org/licenses/by/ $4.0 /)$
1 School of Biological and Environmental Sciences, Shoolini University of Biotechnology and Management Sciences, Solan 173229, India; prmoo267@gmail.com (P.P.); sunilpuri@shooliniuniversity.com (S.P.); surajpandiar75@gmail.com (S.P.); neeruguleria1532001@gmail.com (N.K.); mamtaparmar369@gmail.com (M.T.); soniagunnu1981@gmail.com (S.R.)

2 Chemical and Biochemical Processing Division, ICAR-Central Institute for Research on Cotton Technology, Mumbai 400019, India

3 School of Mechanical and Civil Engineering, Shoolini University of Biotechnology and Management Sciences, Solan 173229, India; ashok.pundir78791@gmail.com

4 Department of Zoology Saradar Vallabhbhai Patel, Cluster University, Mandi 175001, India; Radhika_mnd@yahoo.com

5 MS Swaminathan School of Agriculture, Shoolini University of Biotechnology and Management Sciences, Solan 173229, India; Salenajanjua13@gmail.com (S.J.); mansoorsoil86@gmail.com (M.A.)

6 Department of Food, Nutrition and Packaging Sciences, Clemson University, Clemson, SC 29634, USA; snehpunia69@gmail.com

7 School of Basic and Applied Sciences, Sanskriti University, Mathura 281401, India; cpsingh.2012@gmail.com

8 Department of Veterinary Sciences and Animal Husbandry, Amrita School of Agricultural Sciences, Amrita Vishwa Vidyapeetham University, Coimbatore 642109, India; c_deepak@cb.amrita.edu

9 Department of Agronomy, Amrita School of Agricultural Sciences, Amrita Vishwa Vidyapeetham University, Coimbatore 642109, India; r_sureshkumar@cb.amrita.edu

10 Department of Rural Development and Agricultural Extension, College of Agriculture, Wolaita Sodo University, Wolaita Sodo P.O. Box 138, Ethiopia; drsenapathy@wsu.edu.et

11 Division of Horticulture, RCSM College of Agriculture, Kolhapur 416004, India; sangram1326@hotmail.com

12 Dr. S. S. Bhatnagar University Institute of Chemical Engineering and Technology, Panjab University, Chandigarh 160014, India

13 HCP Division, ICAR - Central Institute of Post-Harvest Engineering and Technology, Abohar 152116, India; Mahesh.samota@icar.gov.in

14 ICAR - National Research Centre on Pomegranate, Solapur 413255, India; rahul.damale@icar.gov.in

15 Division of Crop Physiology, Biochemistry and Post-Harvest Technology, ICAR-Central Potato Research Institute, Shimla 171001, India; sushil.changan@icar.gov.in

16 ICAR-National Research Centre for Orchids, Pakyong 737106, India; nattabiochem@gmail.com

17 Department of Microbiology, College of Medicine, Taif University, P.O. Box 11099, Taif 21944, Saudi Arabia; mabulihd@tu.edu.sa

18 Biology Department, Faculty of Science, King Khalid University, Abha 61421, Saudi Arabia; elkottaf@kku.edu.sa

19 Zoology Department, College of Science, Damanhour University, Damanhour 22511, Egypt

20 Department of Pharmaceutical Sciences, Pharmacy Program, Batterjee Medical College, Jeddah 21442, Saudi Arabia; abdeldaim.m@vet.suez.edu.eg

21 Pharmacology Department, Faculty of Veterinary Medicine, Suez Canal University, Ismailia 41522, Egypt

* Correspondence: radhuchauhan7002@gmail.com (R.); manoj.kumar13@icar.gov.in (M.K.); ssbhinder@pu.ac.in (S.S.)

Abstract: The aim of current study is to provide a significant traditional knowledge on wild medicines used for ethnoveterinary purposes in the rural area of Maraog region in district Shimla. The medicinal plants have played a significant role in the treatment of human as well as animal's diseases. The rural people of the Maraog region were interviewed through a questionnaire and extensive field 
surveys were conducted from June 2020 to July 2021. The discussion, observations and interviews were conducted in study site and included 96 informants. The information gathered from the residents is presented in a table format and includes scientific and local names, different parts used, diseases treated and mode administration. The most commonly used taxa are calculated with used value. The study revealed 100 plants in which trees (7), shrubs (26), herbs (56), ferns (5), grasses (3) and climbers (3) were identified. The most commonly documented livestock diseases were found to be hoof infections, eyes infections, poisoning and skin infections. In the current study, the Rosaceae family was reported as being the highest number (11), followed by Asteraceae (10) and then Lamiaceae (6). It was found that leaves, roots, flowers and fruits are the commonly used parts for ethnoveterinary medications. The phytochemicals present in the plant, such as alkaloids, sterols, glycosides, flavonoids, lignin, coumarins and terpenoids, etc., may be responsible for their medicinal properties. In this documentation, it was observed that the younger generation does not have good knowledge of medicinal plants as compared to the older ones. Therefore, it is necessary to preserve the traditional knowledge of these medicinal plants before their permanent loss. The documentation and conservation of medicinal plants can be a good start for novel phytopharmacological research in the veterinary field.

Keywords: therapeutic uses; ethnoveterinary plants; rural people; livestock

\section{Introduction}

Ethnoveterinary medicine is a branch of science that explores the values, methods, skills, procedures and practices used for animal health care [1-4]. In rural areas of the northwestern Himalaya, plants are important for ethnic communities' survival. As the World Health Organization (WHO) reports, approximately $80 \%$ of the world's population is directly dependent on plant resources for their health, especially those living in rural regions $[5,6]$. India is considered a rich source of biodiversity, with two mega biodiversity hotspots, the Western Ghats and Eastern Himalayas [7]. The state of Himachal Pradesh has about 3256 plant species [8]. There are 3120 species of angiosperms, 124 species of pteridophytes and 12 species of gymnosperms in Himachal Pradesh. The high and low hills are covered with coniferous forests, with oak trees growing in the hollows. Firs and spruce dominate the higher elevations, while pines cover the lower elevations. The Himachal Pradesh is rich source of herbal medicinal plants and people of some areas are completely dependent on these plants for their well-being. In different reports, it was documented that aromatic and medicinal plants are used for various therapeutic purposes [9-11]. It was reported that different plants species have been used in India for medicinal purposes and human nourishment since Vedic times [12]. Medicinal plants possess different types of phytochemicals, such as alkaloids, flavonoid and saponin. [13-15]. The different natural resources from forests such as wild plants are used to fulfil the daily human beings' requirements, such as fuel, fodder and medicines [16,17]. Some wild plants yielding fruits are major source of nutrients and economy for many communities throughout the world $[18,19]$. Since ancient times, plants have been used by the majority of tribal communities. Some causes, such as rising drug prices and veterinary practices, have suddenly increased interest in the field of ethnoveterinary research [20]. People acquire ethnoveterinary information through several years of experience and only orally pass it on from generation to generation (oral tradition). With rapid cultural changes and modernization, this traditional information is being loss [21]. Therefore, there is an urgent need to for scientific documentation of traditional information from rural areas of India [22]. Different types of ethnoveterinary research on the use of plants in therapeutic studies have been conducted around the world [23]. Ethnoveterinary traditional knowledge is applied for the health care system of domestic animals [24,25]. In India, since the Vedic era, plants have been used in veterinary treatment [26-28]. The current study documented a large number of wild plants with medicinal properties and therapeutic applications which are 
still unknown from Maraog region in Shimla district. In this context, it is important to conduct the extensive field surveys in unexplored regions that document the ethnoveterinary knowledge, because rapid urbanization and the greater use of synthetic drugs or medicines and culture variations may contribute to the loss of traditional knowledge from society. In addition, it can also provide important information for the selection of natural alternatives for treating livestock diseases and finding new drugs. Therefore, an attempt has been performed to document some useful indigenous knowledge of ethnoveterinary uses of plants from the rural region of Maraog in district Shimla of Himachal Pradesh, India. It was observed that due to modernization in the society, new generation of the study area is not interested in traditional knowledge; hence, the compilation of these ethnoveterinary plants is need of time. The usefulness of ethnopharmacology for drug development will be severely limited due to a lack of regional knowledge base concerning medicinal plants that can be exploited in veterinary ethnopharmacology. This study was conducted in the Shimla district of Maraog region in order to preserve the traditional knowledge of medicinal plants. The primary aim of this research was to collect and document the local traditional knowledge of ethnoveterinary plants used by native people of study site.

\section{Materials and Methods}

\subsection{Description of the Study Area}

The survey was carried out in Maraog region in tehsil Chopal of Shimla district. Maraog region is a rural area in Shimla district of Himachal Pradesh with a diverse ecological, archaeological, religious, mythological and spiritual community Figure 1. Chopal forest division is divided into 7 forest ranges and has 73 forest beats and 22 forest blocks [29]. Agriculture and farming are the primary occupations of the residents of the district. Apple, maize, green vegetables and potato are the chief cultivation crops grown in the study region. The animals such as sheep, goats, cows and buffalos are popular at the study site and provide meat, milk and milk products for rural people in Shimla district.
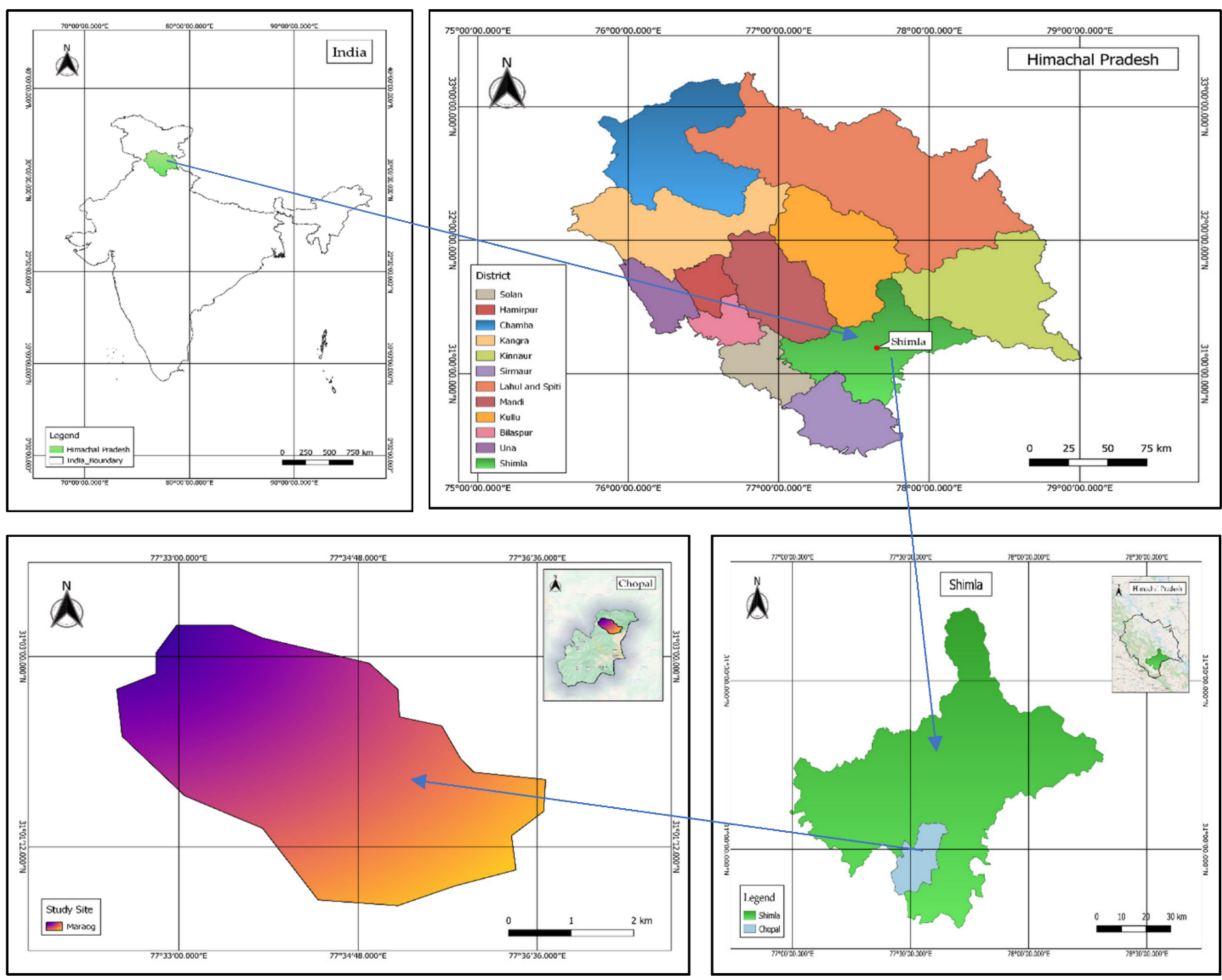

Figure 1. Scale map of study site. 


\subsection{Sampling Informants}

During a survey, a total of 96 informants ( 57 male and 39 female) were selected for interview. The age and educational background of informants were documented during interview. The informants were divided into 5 groups on the basis of their age (Table 1). In this study, it is discovered that aged people have immense traditional knowledge of ethnoveterinary plants as compared to younger generation.

Table 1. Demography and literacy among informants.

\begin{tabular}{|c|c|c|c|c|c|c|}
\hline Sr. No. & Age Groups & \multicolumn{5}{|c|}{ No. of Informants } \\
\hline 1. & $25-36$ & \multicolumn{5}{|c|}{18 (10 Male and 8 Female) } \\
\hline 2. & $37-46$ & \multicolumn{5}{|c|}{25 (15 Male and 10 Female) } \\
\hline 3. & $47-56$ & \multicolumn{5}{|c|}{37 (21 Male and 16 Female) } \\
\hline 4. & $57-66$ & \multicolumn{5}{|c|}{10 (7 Male and 3 Male) } \\
\hline 5. & $67-80$ & \multicolumn{5}{|c|}{6 (4 Male and 2 Female) } \\
\hline \multirow{2}{*}{\multicolumn{2}{|c|}{ Literacy Among Informants: }} & \multicolumn{5}{|c|}{ Age Groups } \\
\hline & & $25-36$ & $37-46$ & $47-56$ & $57-66$ & $67-80$ \\
\hline 1. & $\begin{array}{l}\text { Never attended } \\
\text { school }\end{array}$ & 0 & 0 & $\begin{array}{l}3 \text { (2 Male, } \\
1 \text { Female) }\end{array}$ & $\begin{array}{l}5 \text { (3 Male, } \\
2 \text { Female) }\end{array}$ & $\begin{array}{l}5 \text { (4 Male, } \\
1 \text { Female) }\end{array}$ \\
\hline 2. & $\begin{array}{l}\text { Attended school } \\
\text { up to primary } \\
\text { level (1-5 class) }\end{array}$ & 0 & $\begin{array}{l}5 \text { (2 Male, } \\
3 \text { Female) }\end{array}$ & $\begin{array}{l}2 \text { (1 Male, } \\
1 \text { Female) }\end{array}$ & 1 (1 Male) & 1 (1 Male) \\
\hline 3. & $\begin{array}{l}\text { Attended school } \\
\text { up to middle } \\
\text { level (6-8) }\end{array}$ & 0 & $\begin{array}{l}5 \text { (3 Male, } \\
2 \text { Female) }\end{array}$ & $\begin{array}{l}6 \text { (4 Male, } \\
2 \text { Female) }\end{array}$ & $\begin{array}{l}3 \text { (2 Male, } \\
1 \text { Female) }\end{array}$ & 0 \\
\hline 4. & $\begin{array}{l}\text { Attended school } \\
\text { up to metric level } \\
\text { (9-10 class) }\end{array}$ & $\begin{array}{l}18 \text { (10 Male, } \\
9 \text { Female) }\end{array}$ & $\begin{array}{l}15 \text { (9 Male, } \\
6 \text { Female) }\end{array}$ & $\begin{array}{l}26 \text { (15 Male, } \\
11 \text { Female) }\end{array}$ & 1 (1 Male) & 0 \\
\hline
\end{tabular}

\subsection{Ethnoveterinary Data Collection and Ethical Considerations}

The ethnoveterinary data were collected from Maraog region in district Shimla, Himachal Pradesh India. A total of 96 male and female informants were selected by snowball methods for interviews. The ethnoveterinary data were collected through pretested questionnaire, observation, interviews and participatory observations [30,31]. The extensive field visits were used to collect ethnoveterinary data from unexplored rural area of Maraog in tehsil Chopal of Shimla district, Himachal Pradesh, India. For ethnoveterinary survey questionnaire was divided into 3 sections: demographic data, ethnoveterinary plant uses and informant's declaration. The ethnoveterinary plants species collected from Maraog region in between 2020 to 2021 and they were identified from Botanical Survey of India, Dehradun Uttarakhand, India. The identified plant specimens with voucher numbers were submitted to the herbarium of Shoolini University, Solan, Himachal Pradesh, India.

Questionnaire for Conducting the Ethnoveterinary Study

The authors followed a specific questionnaire for conducting this study, as given in Supplementary Information S1.

\subsection{Data Analysis}

Ethnoveterinary data were collected by snowball method and selected 96 informants (male and female) from Maraog region in tehsil Chopal. The ethnoveterinary data were analyzed through used value.

Use Value

The use value is an ethnobotanical key that has been used to calculate the relative value of useful plant species [32,33]. 


$$
\mathrm{UV}=\Sigma \mathrm{Ui} / \mathrm{n}
$$

where $\mathrm{Ui}$ is the number of usage reports cited by each informer for a given plants and $\mathrm{n}$ is total number of informants. The use value is important to find which plants are most useful to specific inhabitants, estimating possible medicinal plant uses and determining community awareness $[34,35]$. It has been mentioned that use value places more significance to plants which have various uses, even if these species are not well identified [36]. A high use value indicates that the plant is important, while a low or zero use value indicates that the plant is rarely used or recorded. There is no indication in the use value whether the plant is used for one or more purposes [37].

\section{Results}

\subsection{Ethnoveterinary Plants Reported by the Informants}

In study site a total of 96 informants reported 100 plant species used for ethnoveterinary purposes. In this study, it was found that Rosaceae, Asteraceae and Lamiaceae were the highest reported families by rural people of Maraog. The Rosaceae family had 11 plant species, followed by Asteraceae family with 10 plants and the Lamiaceae family with 6 plants; the Apiaceae, Brassicaceae and Solanaceae families each contributed 3 plant species, while the Fabaceae and Polygonaceae families each contributed 4 plant species; the Amaranthaceae, Berberidaceae, Oxalidaceae, Pinaceae, Poaceae, Primulaceae, Pteridaceae, Ranunculaceae, Scrophulariaceae and Utricaceae contributed 2 plant species (Figure 2).

Some ethnomedicinal plants in the study area are well known for ethnoveterinary purpose was found as Chenopodium album, Cannabis sativa, Cynodon dactylon, Bromus hordeaceus, Cedrus deodara, Ajuga parviflora, Foeniculum vulgare, Sonchus oleraceus and Urtica dioica. The documented plants include 56 herbs, 26 shrubs, 7 trees, 5 ferns, 3 climbers and 3 grasses (Figure 3).

As shown in Figure 4, the rhizome, tubers and buds were the least frequently documented plant parts used in ethnoveterinary purpose.

\subsection{Use Value of Medicinal Plants}

It was found that few plant species have greater used value Cannabis sativa (0.79), Cynodon dactylon (0.75), Datura stramonium (0.71), Rhododendron arboreum (0.71), Chenopodium album (0.69), Hedera nepalensis (0.68), Mentha viridis (0.65) and Viola canescens (0.63).

Different plant parts roots, leaves, stem, rhizoids and tubers, etc., are used to treat a variety of livestock diseases. A total of 100 plants were documented for ethnoveterinary preparations in rural region of Maraog. In discussion with aged people, they reported skin infections, mouth blisters, diarrhea, gastrointestinal disorders, cuts, udder infections and eye disorders were the most commonly found symptoms in livestock. The most popular methods of preparation include the use of leaf paste, infusion and decoction, with other methods such as wood ash being recorded less frequently. According to the data collected from the informants, it was found that the mode of administration can be oral or topical and certain plants can be used both orally and topically to treat livestock diseases. The majority of preparations use a single plant, with just a few combining multiple plant species.

Table 2 describes all plants reported with their botanical name, family, habit, parts used and ailment treated. 


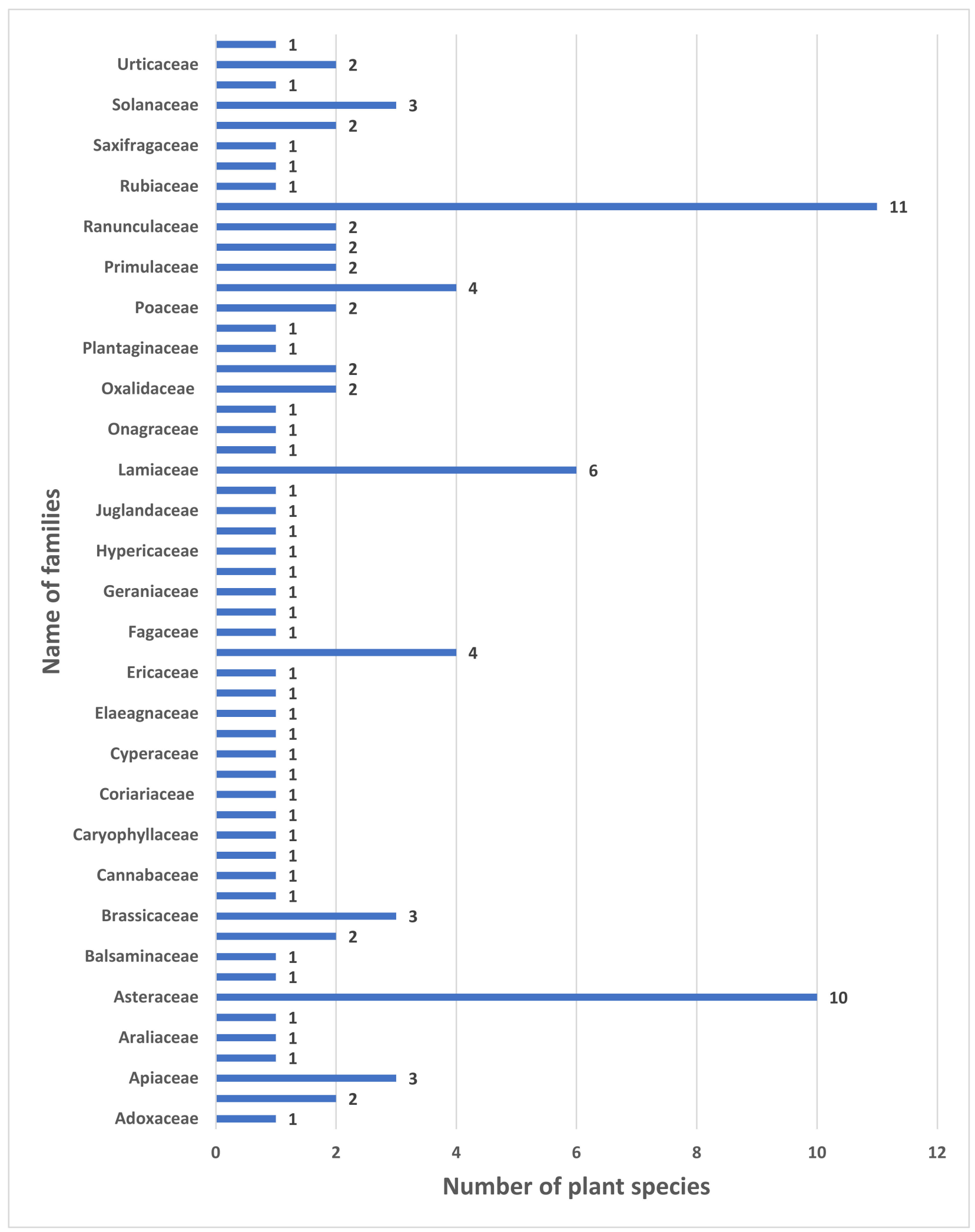

Figure 2. Representation of the families and number of plants studied at study site. 


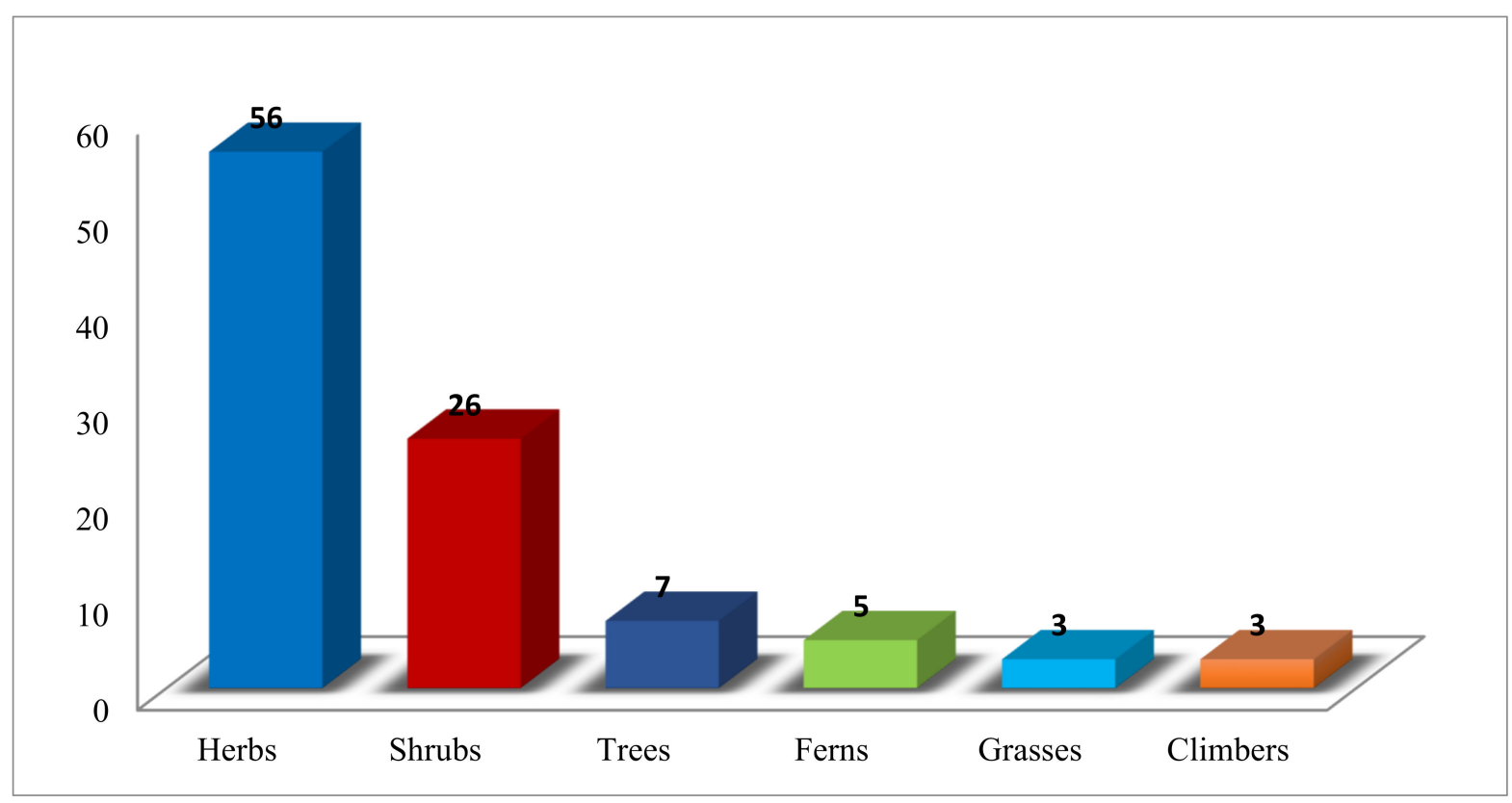

Figure 3. Growth forms of plant species at study site.

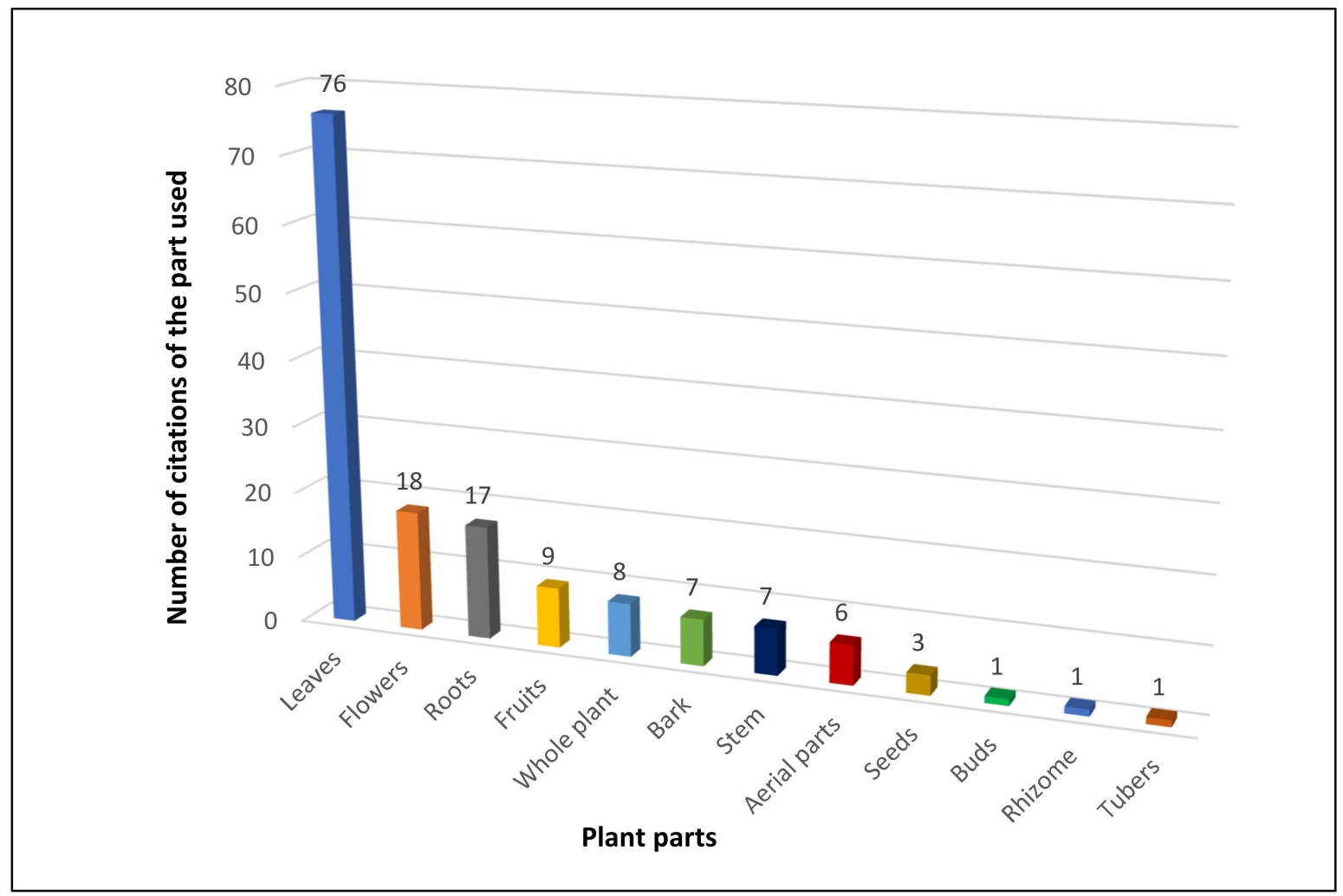

Figure 4. Representation of the number of citations of plant parts used. 
Table 2. Ethnomedicinal plants used in study area.

\begin{tabular}{|c|c|c|c|c|c|c|c|c|c|c|}
\hline Botanical Name & $\begin{array}{l}\text { Actual Morphology of } \\
\text { Ethnomedicinal Plant }\end{array}$ & Family & Common Name & Voucher No. & Habit & Parts Used & Disease Treated & Mode & $\begin{array}{c}\text { Ailment Treated and } \\
\text { Citations }\end{array}$ & Use-Value \\
\hline $\begin{array}{l}\text { Abies pindrow (Royle ex } \\
\text { D. Don) Royle }\end{array}$ & & Pinaceae & Tonss & SUBMS/BOT-4184 & Tree & Leaves & Udder infection & Topical & $\begin{array}{l}\text { Leaf paste is applied on } \\
\text { udder of cow to treat } \\
\text { clotting of milk (66). }\end{array}$ & 0.68 \\
\hline $\begin{array}{l}\text { Adiantum venustum } \mathrm{D} . \\
\text { Don }\end{array}$ & & Pteridaceae & Jamna & SUBMS/BOT-4185 & Fern & Leaves & Skin disease & Topical & $\begin{array}{l}\text { Paste of plant part is } \\
\text { applied on chronic tumors } \\
\text { for rapid healing (57). }\end{array}$ & 0.59 \\
\hline Ajuga parviflora Benth. & & Lamiaceae & Neel kanthi & SUBMS/BOT-4186 & Herb & Aerial part & Sores, Wounds & Topical & $\begin{array}{l}\text { Fine powder of aerial parts } \\
\text { with few drops of edible oil } \\
\text { is applied on skin of cattle } \\
\text { to treat sores, wounds (69). }\end{array}$ & 0.71 \\
\hline Amaranthus, blitum $\mathrm{L}$. & & Amaranthaceae & Sukhichalayi & SUBMS/BOT-4187 & Herb & Leaves & $\begin{array}{l}\text { Diarrhea, } \\
\text { Dysentery, } \\
\text { Skin infection }\end{array}$ & Oral, Topical & $\begin{array}{l}\text { Fresh leaves are used to } \\
\text { cure diarrhea and dysentery. } \\
\text { Paste of leaves is useful in } \\
\text { curing skin infection (48). }\end{array}$ & 0.50 \\
\hline
\end{tabular}


Table 2. Cont.

\begin{tabular}{|c|c|c|c|c|c|c|c|c|c|c|}
\hline Botanical Name & $\begin{array}{l}\text { Actual Morphology of } \\
\text { Ethnomedicinal Plant }\end{array}$ & Family & Common Name & Voucher No. & Habit & Parts Used & Disease Treated & Mode & $\begin{array}{c}\text { Ailment Treated and } \\
\text { Citations }\end{array}$ & Use-Value \\
\hline $\begin{array}{l}\text { Androsace sarmentosa } \\
\text { Wall. }\end{array}$ & & Primulaceae & Phoolru & SUBMS/BOT-4188 & Herb & Leaves & Skin infections & Topical & $\begin{array}{l}\text { Paste of fresh leaves is } \\
\text { applied on skin infections } \\
\text { (45). }\end{array}$ & 0.46 \\
\hline $\begin{array}{l}\text { Artemisia vestita Wall. } \\
\text { Ex Besser }\end{array}$ & & Asteraceae & Chamber & SUBMS/BOT-4189 & Herb & $\begin{array}{l}\text { Leaves, } \\
\text { Flowers }\end{array}$ & Wounds & Topical & $\begin{array}{l}\text { Paste of leaves or flowers is } \\
\text { used to treat wounds (54). }\end{array}$ & 0.56 \\
\hline $\begin{array}{c}\text { Aruncus dioicus (Walter) } \\
\text { Fernald }\end{array}$ & & Rosaceae & Pothee & SUBMS/BOT-4190 & Herb & Roots & $\begin{array}{l}\text { Internal bleeding, } \\
\text { Stomach pains, } \\
\text { Diarrhea }\end{array}$ & Oral & $\begin{array}{l}\text { Roots along with warm } \\
\text { water is used to stop } \\
\text { bleeding after birth } \\
\text { Paste of fresh roots cures } \\
\text { stomachache and diarrhea } \\
(56) \text {. }\end{array}$ & 0.58 \\
\hline $\begin{array}{l}\text { Asplenium dalhousiae } \\
\text { Hook. }\end{array}$ & & Aspleniaceae & Nanwein & SUBMS/BOT-4191 & Fern & Whole plant & Skin infection & Topical & $\begin{array}{l}\text { Whole plant is used to treat } \\
\text { bacterial skin infections } \\
\text { (48). }\end{array}$ & 0.50 \\
\hline
\end{tabular}


Table 2. Cont

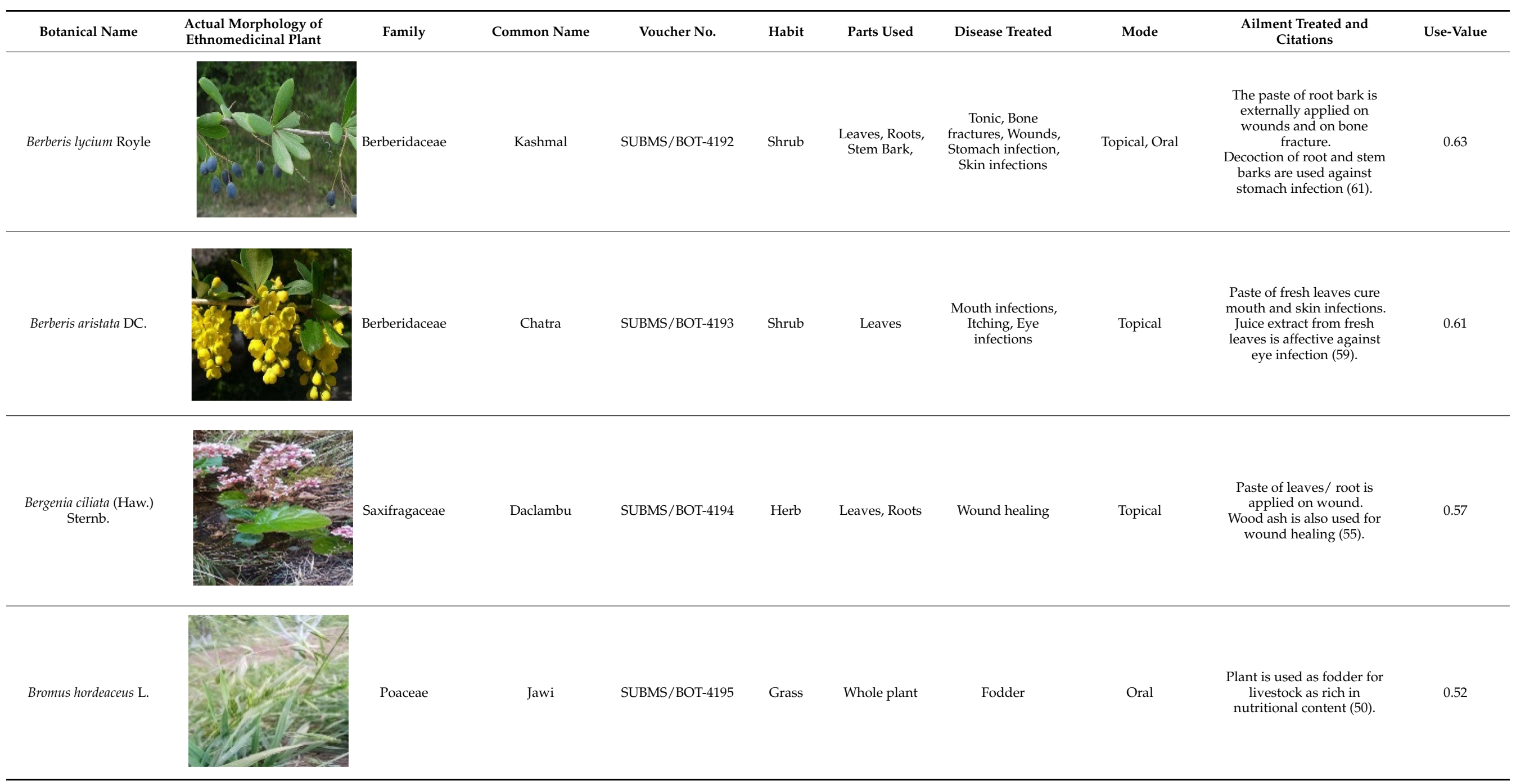


Table 2. Cont

\begin{tabular}{|c|c|c|c|c|c|c|c|c|c|c|}
\hline Botanical Name & $\begin{array}{l}\text { Actual Morphology of } \\
\text { Ethnomedicinal Plant }\end{array}$ & Family & Common Name & Voucher No. & Habit & Parts Used & Disease Treated & Mode & $\begin{array}{c}\text { Ailment Treated and } \\
\text { Citations }\end{array}$ & Use-Value \\
\hline Buddleja crispa Benth. & & Scrophulariaceae & Taakla & SUBMS/BOT-4196 & Shrub & Leaves & $\begin{array}{l}\text { Cold, Dysentery, } \\
\text { Bleeding }\end{array}$ & Oral, Topical & $\begin{array}{l}\text { Fresh leaves are given to } \\
\text { animals to cure cold and } \\
\text { dysentery. } \\
\text { Paste of fresh leaves is used } \\
\text { to stop bleeding (47). }\end{array}$ & 0.48 \\
\hline Cannabis sativa $\mathrm{L}$. & & Cannabaceae & Bhang & SUBMS/BOT-4197 & Herb & Leaves & $\begin{array}{l}\text { Intestinal worms, } \\
\text { Stomach pain }\end{array}$ & Oral & $\begin{array}{l}\text { Powdered leaf balls are } \\
\text { given to cattle to treat } \\
\text { intestinal worms and body } \\
\text { pain (76). }\end{array}$ & 0.79 \\
\hline $\begin{array}{l}\text { Capsella bursa-pastoris } \\
\text { (L.) Medik. }\end{array}$ & & Brassicaceae & Khandwa & SUBMS/BOT-4198 & Herb & Leaves & Wound & Topical & $\begin{array}{l}\text { Leaf paste is used to cure } \\
\text { wounds (54). }\end{array}$ & 0.56 \\
\hline $\begin{array}{l}\text { Cedrus deodara (Roxb. } \\
\text { ex D.Don) G. Don }\end{array}$ & & Pinaceae & Devdar & SUBMS/BOT-4199 & Tree & Leaves & Skin infections & Topical & $\begin{array}{l}\text { Paste of fresh leaves is } \\
\text { applied on skin to cure } \\
\text { infections (53). }\end{array}$ & 0.55 \\
\hline
\end{tabular}


Table 2. Cont

\begin{tabular}{|c|c|c|c|c|c|c|c|c|c|c|}
\hline Botanical Name & $\begin{array}{l}\text { Actual Morphology of } \\
\text { Ethnomedicinal Plant }\end{array}$ & Family & Common Name & Voucher No. & Habit & Parts Used & Disease Treated & Mode & $\begin{array}{c}\text { Ailment Treated and } \\
\text { Citations }\end{array}$ & Use-Value \\
\hline $\begin{array}{l}\text { Cirsium arvense (L.) } \\
\text { Scop. }\end{array}$ & & Asteraceae & Bhenda & SUBMS/BOT-4201 & Herb & Whole plant & Digestion & Oral & $\begin{array}{l}\text { Used as feed for ruminants } \\
\text { due to high nutritional } \\
\text { value (45). }\end{array}$ & 0.46 \\
\hline $\begin{array}{l}\text { Clematis buchananiana } \\
\text { DC. }\end{array}$ & & Ranunculaceae & Silra & SUBMS/BOT-4202 & Climber & Whole plant & Wound healing & Topical & $\begin{array}{l}\text { Plant paste is applied on } \\
\text { wounds (56). }\end{array}$ & 0.58 \\
\hline Clematis vitalba $\mathrm{L}$. & & Ranunculaceae & Garol & SUBMS/BOT-4203 & Shrub & Leaves & Skin eruptions & Topical & $\begin{array}{l}\text { Paste of leaves is applied on } \\
\text { skin eruptions in livestock } \\
\text { (47). }\end{array}$ & 0.48 \\
\hline
\end{tabular}


Table 2. Cont.

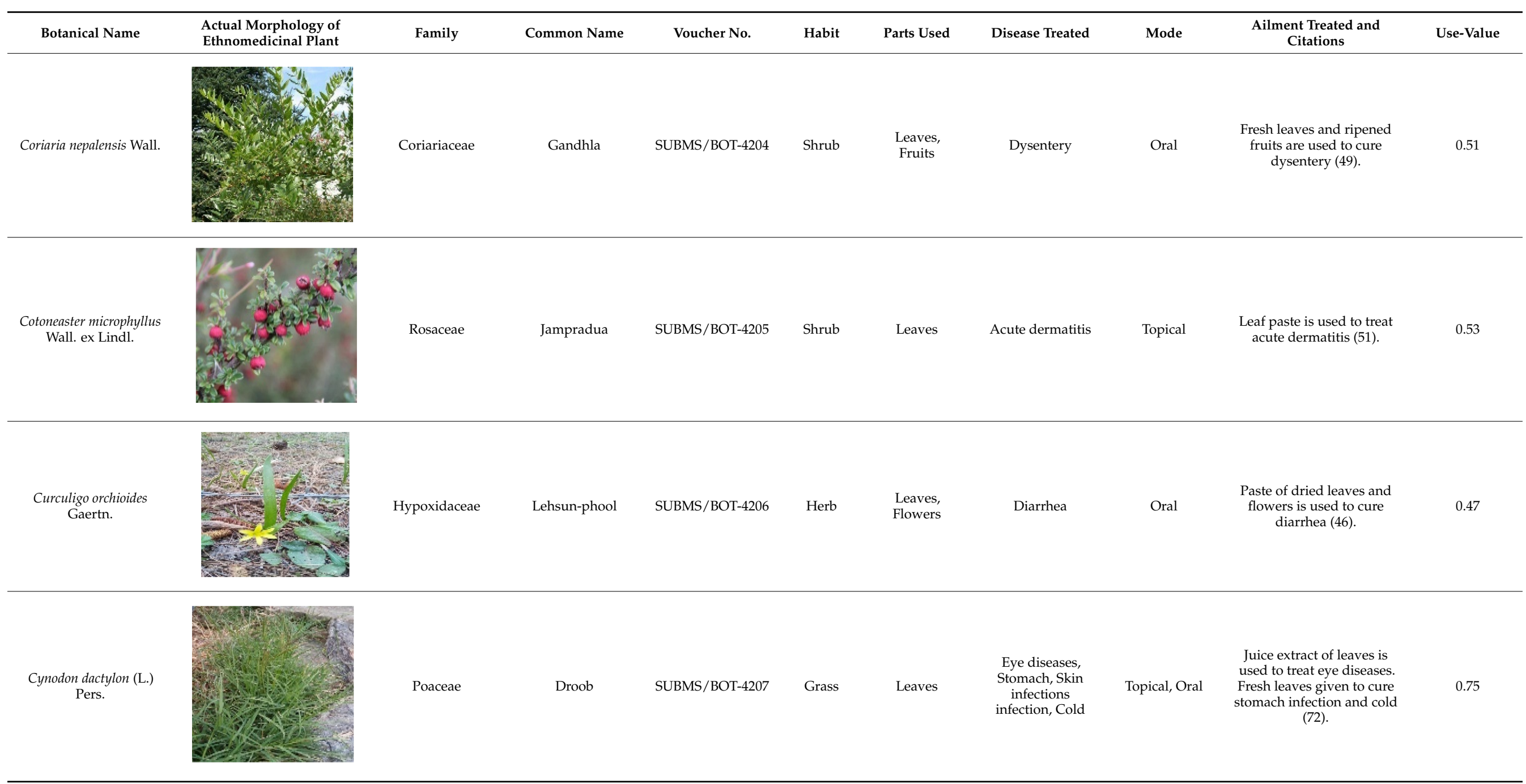


Table 2. Cont.

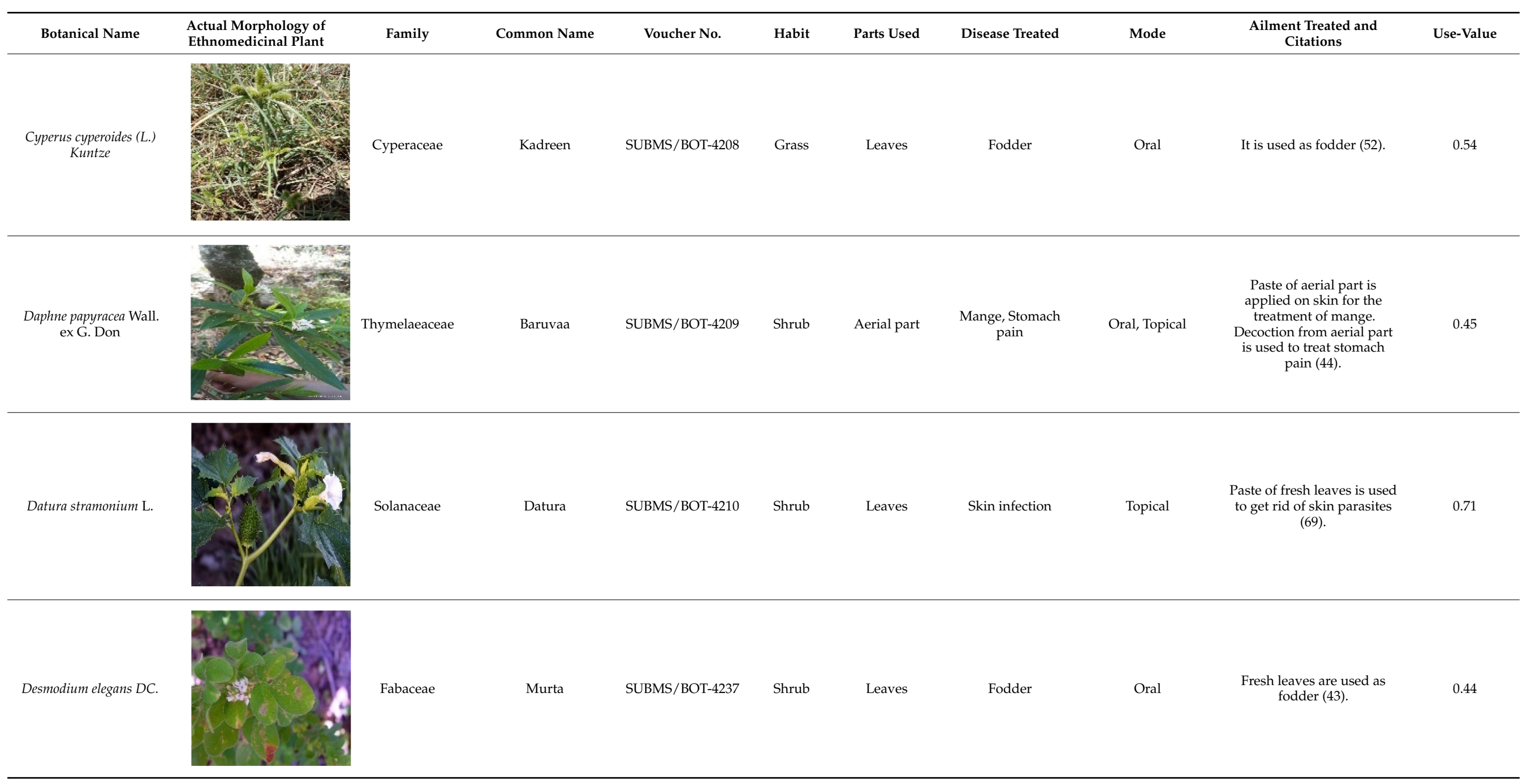


Table 2. Cont

\begin{tabular}{|c|c|c|c|c|c|c|c|c|c|c|}
\hline Botanical Name & $\begin{array}{l}\text { Actual Morphology of } \\
\text { Ethnomedicinal Plant }\end{array}$ & Family & Common Name & Voucher No. & Habit & Parts Used & Disease Treated & Mode & $\begin{array}{l}\text { Ailment Treated and } \\
\text { Citations }\end{array}$ & Use-Value \\
\hline Deutzia scabra Thunb. & & Hydrangeaceae & Suniya & SUBMS/BOT-4211 & Shrub & Leaves & Skin infections & Topical & $\begin{array}{l}\text { Paste of fresh leaves is } \\
\text { useful against skin } \\
\text { infections (51). }\end{array}$ & 0.53 \\
\hline $\begin{array}{l}\text { Diplazium esculentum } \\
\text { (Retz.) Sw }\end{array}$ & & Athyriaceae & Lingar & SUBMS/BOT-4239 & Fern & Leaves & Diarrhea & Oral & $\begin{array}{l}\text { Young leaves boiled in } \\
\text { water is useful against } \\
\text { diarrhea ( } 39) .\end{array}$ & 0.40 \\
\hline $\begin{array}{l}\text { Elaeagnus umbellata } \\
\text { Thunb. }\end{array}$ & & Elaeagnaceae & Genhi & SUBMS/BOT-4212 & Shrub & Flowers & $\begin{array}{c}\text { Reduce } \\
\text { inflammation }\end{array}$ & Topical & $\begin{array}{l}\text { Flowers are used to reduce } \\
\text { inflammation on affected } \\
\text { areas (44). }\end{array}$ & 0.45 \\
\hline Epilobium hirsutum $\mathrm{L}$. & & Onagraceae & Dandri & SUBMS/BOT-4213 & Herb & Leaves & $\begin{array}{l}\text { Mouth ulcers, } \\
\text { Wasp sting }\end{array}$ & Oral, Topical & $\begin{array}{l}\text { Paste of leaves is used to } \\
\text { treat mouth ulcers and } \\
\text { wasp stings (42). }\end{array}$ & 0.43 \\
\hline
\end{tabular}


Table 2. Cont.

\begin{tabular}{|c|c|c|c|c|c|c|c|c|c|c|}
\hline Botanical Name & $\begin{array}{l}\text { Actual Morphology of } \\
\text { Ethnomedicinal Plant }\end{array}$ & Family & Common Name & Voucher No. & Habit & Parts Used & Disease Treated & Mode & $\begin{array}{l}\text { Ailment Treated and } \\
\text { Citations }\end{array}$ & Use-Value \\
\hline Erigeron alpinus $\mathrm{L}$. & & Asteraceae & Chipru & SUBMS/BOT-4215 & Herb & Leaves & Bleeding & Topical & $\begin{array}{c}\text { Paste of fresh leaves is } \\
\text { applied on bleeding wound } \\
(40) .\end{array}$ & 0.41 \\
\hline Erigeron bonariensis $\mathrm{L}$. & & Asteraceae & Kupru & SUBMS/BOT-4216 & Herb & Leaves & $\begin{array}{l}\text { Stomach pain, } \\
\text { Urinary infection }\end{array}$ & Oral & $\begin{array}{l}\text { Plant is used to treat } \\
\text { stomach pain and urinary } \\
\text { infection (47). }\end{array}$ & 0.48 \\
\hline $\begin{array}{l}\text { Evolvulus nummularius } \\
\text { (L.) L. }\end{array}$ & & Convolvulaceae & Ghareu & SUBMS/BOT-4217 & Herb & Leaves & Skin infection & Topical & $\begin{array}{l}\text { Paste of fresh as well as dry } \\
\text { leaves is applied to cure } \\
\text { skin infections caused by } \\
\text { parasites (53). }\end{array}$ & 0.55 \\
\hline
\end{tabular}


Table 2. Cont

\begin{tabular}{|c|c|c|c|c|c|c|c|c|c|c|}
\hline Botanical Name & $\begin{array}{l}\text { Actual Morphology of } \\
\text { Ethnomedicinal Plant }\end{array}$ & Family & Common Name & Voucher No. & Habit & Parts Used & Disease Treated & Mode & $\begin{array}{c}\text { Ailment Treated and } \\
\text { Citations }\end{array}$ & Use-Value \\
\hline $\begin{array}{c}\text { Fagopyrum acutatum } \\
\text { (Lehm.) Mansf. ex K. } \\
\text { Hammer }\end{array}$ & & Polygonaceae & Fafri & SUBMS/BOT-4218 & Herb & Leaves & Skin infections & Topical & $\begin{array}{l}\text { Paste of leaves is applied on } \\
\text { skin infections (45). }\end{array}$ & 0.46 \\
\hline Foeniculum vulgare Mill. & & Apiaceae & Sounph & SUBMS/BOT-4219 & Herb & Leaves, Seeds & $\begin{array}{l}\text { Poisoning, Cough, } \\
\text { Tonic, Skin } \\
\text { infection }\end{array}$ & Oral, Topical & $\begin{array}{c}\text { Dried seeds are given to } \\
\text { cure poisoning and cough. } \\
\text { Seeds with hot water is } \\
\text { given to animals after } \\
\text { parturition, used as tonic. } \\
\text { (59). }\end{array}$ & 0.61 \\
\hline Fragaria virginiana Mill. & & Rosaceae & Bhumbal & SUBMS/BOT-4220 & Herb & $\begin{array}{l}\text { Roots, Leaves, } \\
\text { Fruits }\end{array}$ & $\begin{array}{l}\text { Skin infection, } \\
\text { Indigestion }\end{array}$ & Oral, Topical & $\begin{array}{l}\text { Juice of ripened fruit is } \\
\text { applied on skin infection. } \\
\text { Paste of powdered root and } \\
\text { leaves is applied on cuts } \\
\text { and wounds (48). }\end{array}$ & 0.50 \\
\hline $\begin{array}{l}\text { Galinsoga quadriradiata } \\
\text { Ruiz \& Pav. }\end{array}$ & & Asteraceae & Sheliya & SUBMS/BOT-4221 & Herb & Leaves & Cuts, Wounds & Topical & $\begin{array}{l}\text { Paste of dry as well as fresh } \\
\text { leaves is applied on cuts } \\
\text { and wounds (47). }\end{array}$ & 0.48 \\
\hline
\end{tabular}


Table 2. Cont

\begin{tabular}{|c|c|c|c|c|c|c|c|c|c|c|}
\hline Botanical Name & $\begin{array}{l}\text { Actual Morphology of } \\
\text { Ethnomedicinal Plant }\end{array}$ & Family & Common Name & Voucher No. & Habit & Parts Used & Disease Treated & Mode & $\begin{array}{c}\text { Ailment Treated and } \\
\text { Citations }\end{array}$ & Use-Value \\
\hline $\begin{array}{c}\text { Gentiana argentea } \\
\text { (Royle ex D.Don) Royle } \\
\text { ex D.Don }\end{array}$ & & Gentianaceae & Bhuin neem & SUBMS/BOT-4222 & Herb & $\begin{array}{l}\text { Leaves, } \\
\text { Flowers, } \\
\text { Roots }\end{array}$ & $\begin{array}{l}\text { Skin infections, } \\
\text { Wound, Poisoning }\end{array}$ & Topical, Oral & $\begin{array}{l}\text { Paste of fresh leaves are } \\
\text { used to treat skin infections } \\
\text { and wounds. } \\
\text { Decoction of whole plant is } \\
\text { used in the treatment of } \\
\text { poisoning (51). }\end{array}$ & 0.53 \\
\hline $\begin{array}{l}\text { Geranium nepalense } \\
\text { Sweet }\end{array}$ & & Geraniaceae & Bhrago-ro-naush & SUBMS/BOT-4223 & Herb & Leaves & Skin infections & Topical & $\begin{array}{l}\text { Paste of fresh leaves is used } \\
\text { to cure skin infections (49). }\end{array}$ & 0.51 \\
\hline $\begin{array}{l}\text { Girardinia diversifolia } \\
\text { (Link) Friis }\end{array}$ & & Urticaceae & Lindu bhaber & SUBMS/BOT-4224 & Shrub & Leaves & Gastric infection & Oral & $\begin{array}{l}\text { Fresh leaves boiled with hot } \\
\text { water is used for treating } \\
\text { gastric infection (47). }\end{array}$ & 0.48 \\
\hline $\begin{array}{c}\text { Goodyera repens (L.) } \\
\text { R.Br. }\end{array}$ & & Orchidaceae & Kaligatti & SUBMS/BOT-4225 & Herb & Leaves, Roots & Stomach pain & Oral & $\begin{array}{l}\text { Powdered leaves and roots } \\
\text { are used to treat stomach } \\
\text { pain (55). }\end{array}$ & 0.57 \\
\hline
\end{tabular}


Table 2. Cont.

\begin{tabular}{|c|c|c|c|c|c|c|c|c|c|c|}
\hline Botanical Name & $\begin{array}{l}\text { Actual Morphology of } \\
\text { Ethnomedicinal Plant }\end{array}$ & Family & Common Name & Voucher No. & Habit & Parts Used & Disease Treated & Mode & $\begin{array}{l}\text { Ailment Treated and } \\
\text { Citations }\end{array}$ & Use-Value \\
\hline $\begin{array}{l}\text { Hedera nepalensis } \\
\text { K.Koch }\end{array}$ & & Araliaceae & Kanewari & SUBMS/BOT-4226 & Climber & Leaves & Leeches & Topical & $\begin{array}{l}\text { Leaf extract is used to } \\
\text { remove leeches in sheep } \\
\text { (66). }\end{array}$ & 0.68 \\
\hline $\begin{array}{l}\text { Helichrysum arenarium } \\
\text { (L.) Moench }\end{array}$ & & Asteraceae & Dhareri & SUBMS/BOT-4227 & Herb & Aerial part & Cough & Oral & $\begin{array}{l}\text { Extract obtained from the } \\
\text { plant is used to treat cough } \\
(48) .\end{array}$ & 0.5 \\
\hline $\begin{array}{l}\text { Heracleum maximum } \mathrm{W} . \\
\text { Bartram }\end{array}$ & & Apiaceae & Patla & SUBMS/BOT-4228 & Herb & Roost & Swellings, Blisters & Topical & $\begin{array}{l}\text { Paste prepared from the } \\
\text { root is applied on swellings } \\
\text { and blisters (43). }\end{array}$ & 0.44 \\
\hline Hypericum perforatum $\mathrm{L}$. & & Hypericaceae & Choli phulya & SUBMS/BOT-4229 & Shrub & Aerial part & Relive pain & Oral & $\begin{array}{l}\text { Used to relive nerve pain } \\
\text { due to puncture wounds } \\
\text { (57). }\end{array}$ & 0.59 \\
\hline
\end{tabular}


Table 2. Cont

\begin{tabular}{|c|c|c|c|c|c|c|c|c|c|c|}
\hline Botanical Name & $\begin{array}{l}\text { Actual Morphology of } \\
\text { Ethnomedicinal Plant }\end{array}$ & Family & Common Name & Voucher No. & Habit & Parts Used & Disease Treated & Mode & $\begin{array}{c}\text { Ailment Treated and } \\
\text { Citations }\end{array}$ & Use-Value \\
\hline Ilex dipyrena Wall. & & Aquifoliaceae & Khareu & SUBMS/BOT-4230 & Tree & Leaves & $\begin{array}{l}\text { Enhance milk } \\
\text { production }\end{array}$ & Oral & $\begin{array}{l}\text { Fresh leaves are fed to cattle } \\
\text { to enhance the milk } \\
\text { secretion and to increase } \\
\text { strength (61). }\end{array}$ & 0.63 \\
\hline $\begin{array}{l}\text { Impatiens glandulifera } \\
\text { Royle }\end{array}$ & & Balsaminaceae & Rdheu & SUBMS/BOT-4231 & Herb & $\begin{array}{l}\text { Leaves, } \\
\text { Flowers }\end{array}$ & Poisoning & Oral & $\begin{array}{l}\text { Infusion of fresh leaves and } \\
\text { flowers is used to cure } \\
\text { poisoning occurred from } \\
\text { toxic plants (59). }\end{array}$ & 0.61 \\
\hline $\begin{array}{l}\text { Indigofera gerardiana } \\
\text { Baker }\end{array}$ & & Fabaceae & Kathi & SUBMS/BOT-4232 & Shrub & Bark & Broken bones & Topical & $\begin{array}{l}\text { Bark is boiled in milk and a } \\
\text { bandage is formed and is } \\
\text { used externally to treat } \\
\text { broken bones ( } 47) \text {. }\end{array}$ & 0.48 \\
\hline Juglans regia $\mathrm{L}$. & & Juglandaceae & Akhrot, khodh & SUBMS/BOT-4233 & Tree & Bark & Oral diseases & Topical & $\begin{array}{l}\text { Paste of bark is used to cure } \\
\text { oral diseases (54). }\end{array}$ & 0.56 \\
\hline
\end{tabular}


Table 2. Cont

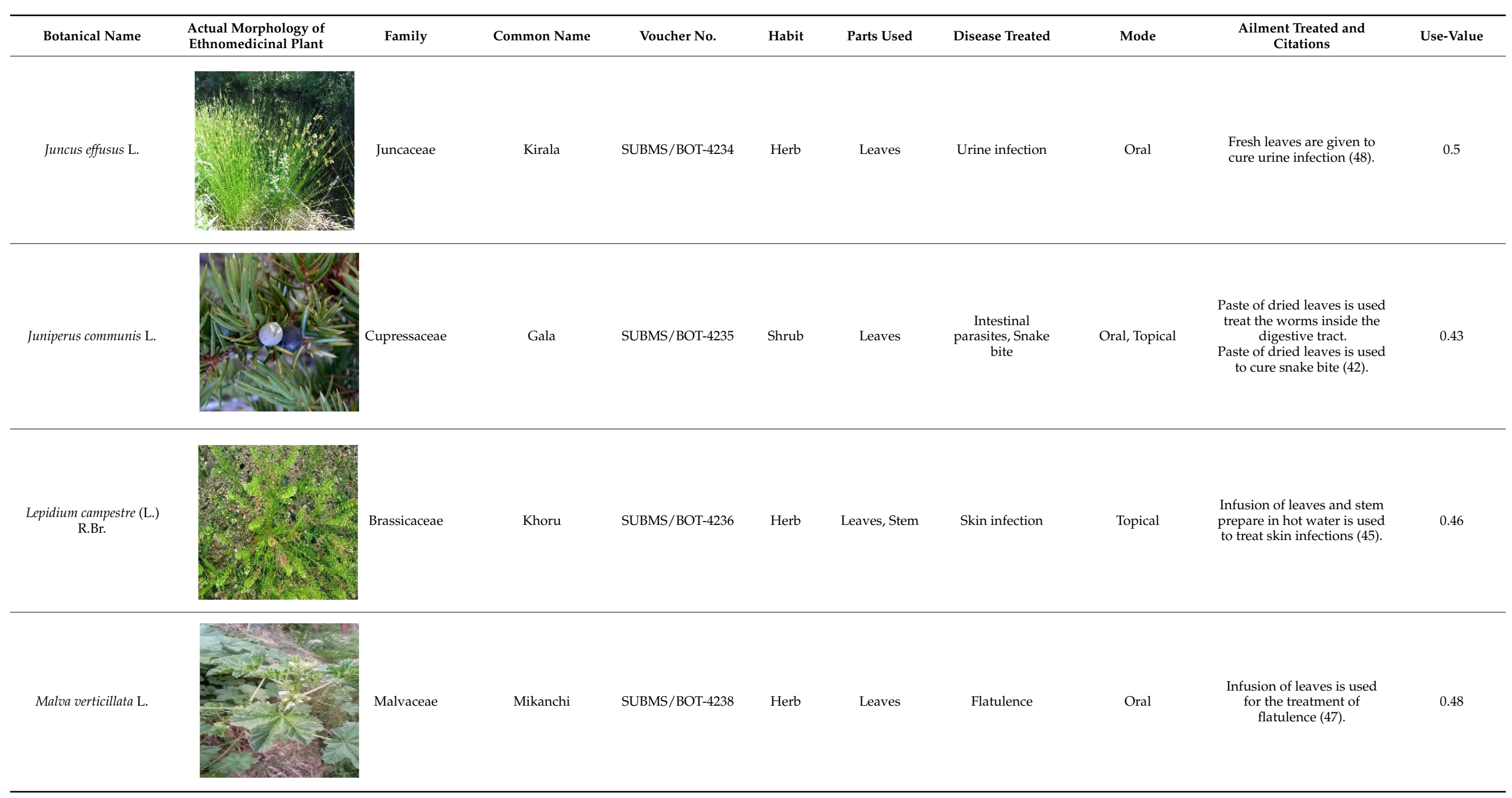


Table 2. Cont

\begin{tabular}{|c|c|c|c|c|c|c|c|c|c|c|}
\hline Botanical Name & $\begin{array}{l}\text { Actual Morphology of } \\
\text { Ethnomedicinal Plant }\end{array}$ & Family & Common Name & Voucher No. & Habit & Parts Used & Disease Treated & Mode & $\begin{array}{c}\text { Ailment Treated and } \\
\text { Citations }\end{array}$ & Use-Value \\
\hline Mentha viridis (L.) L. & & Lamiaceae & Pahari pudina & SUBMS/BOT-4241 & Herb & Leaves & $\begin{array}{l}\text { Stomach infection, } \\
\text { Skin infections, } \\
\text { Hoof diseases }\end{array}$ & Oral, Topical & $\begin{array}{c}\text { Fresh leaves are given to } \\
\text { cure stomach infection. } \\
\text { Paste of fresh leaves is } \\
\text { beneficial against skin } \\
\text { infection and hoof diseases } \\
\text { (63). }\end{array}$ & 0.65 \\
\hline Nicotiana tabacum $\mathrm{L}$. & & Solanaceae & Tambakhoo & SUBMS/BOT-4240 & Herb & Leaves, Stem & Pain relief & Oral & $\begin{array}{l}\text { Decoction of leaves is used } \\
\text { to reduce pain (50). }\end{array}$ & 0.52 \\
\hline $\begin{array}{l}\text { Oxalis articulata } \\
\text { Savigny }\end{array}$ & & Oxalidaceae & Shash & SUBMS/BOT-4242 & Herb & $\begin{array}{l}\text { Leaves, } \\
\text { Flowers }\end{array}$ & Poisoning, Cold & Topical & $\begin{array}{l}\text { Paste of fresh leaves is used } \\
\text { to treat poisoning in animal. } \\
\text { Whole plant is given to cure } \\
\text { cold (47). }\end{array}$ & 0.48 \\
\hline Oxalis corniculata $\mathrm{L}$. & & Oxalidaceae & Shash & SUBMS/BOT-4243 & Shrub & $\begin{array}{l}\text { Leaves, } \\
\text { Flowers }\end{array}$ & Snakebites, Cold & Topical, Oral & $\begin{array}{c}\text { Paste of fresh leaves is used } \\
\text { to treat snakebites. } \\
\text { Whole plant is given to cure } \\
\text { cold (52). }\end{array}$ & 0.54 \\
\hline
\end{tabular}


Table 2. Cont

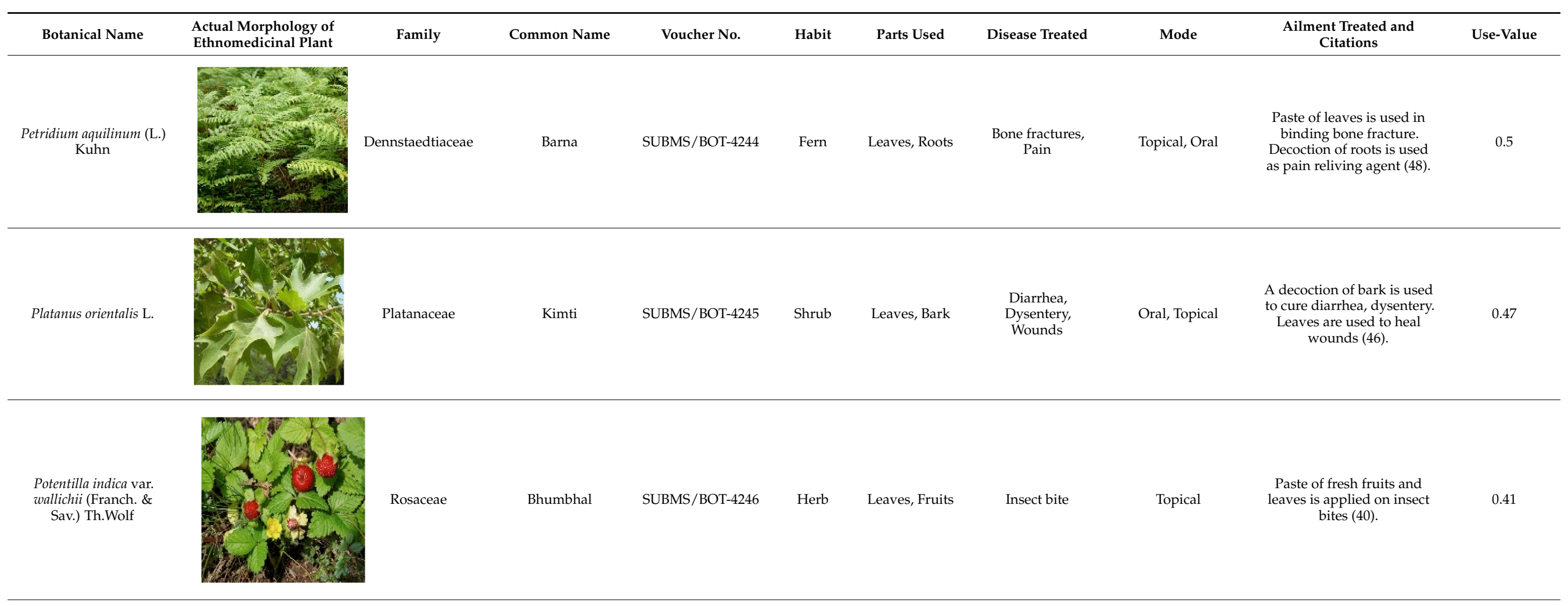

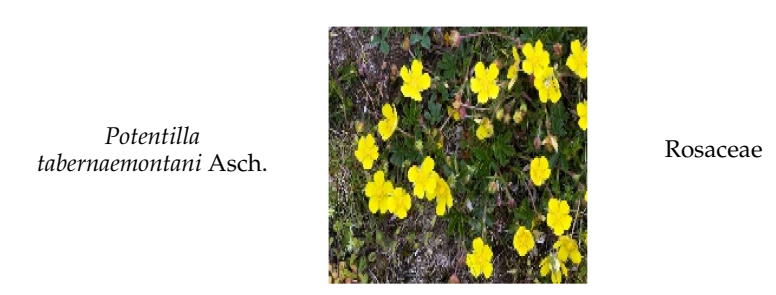


Table 2. Cont

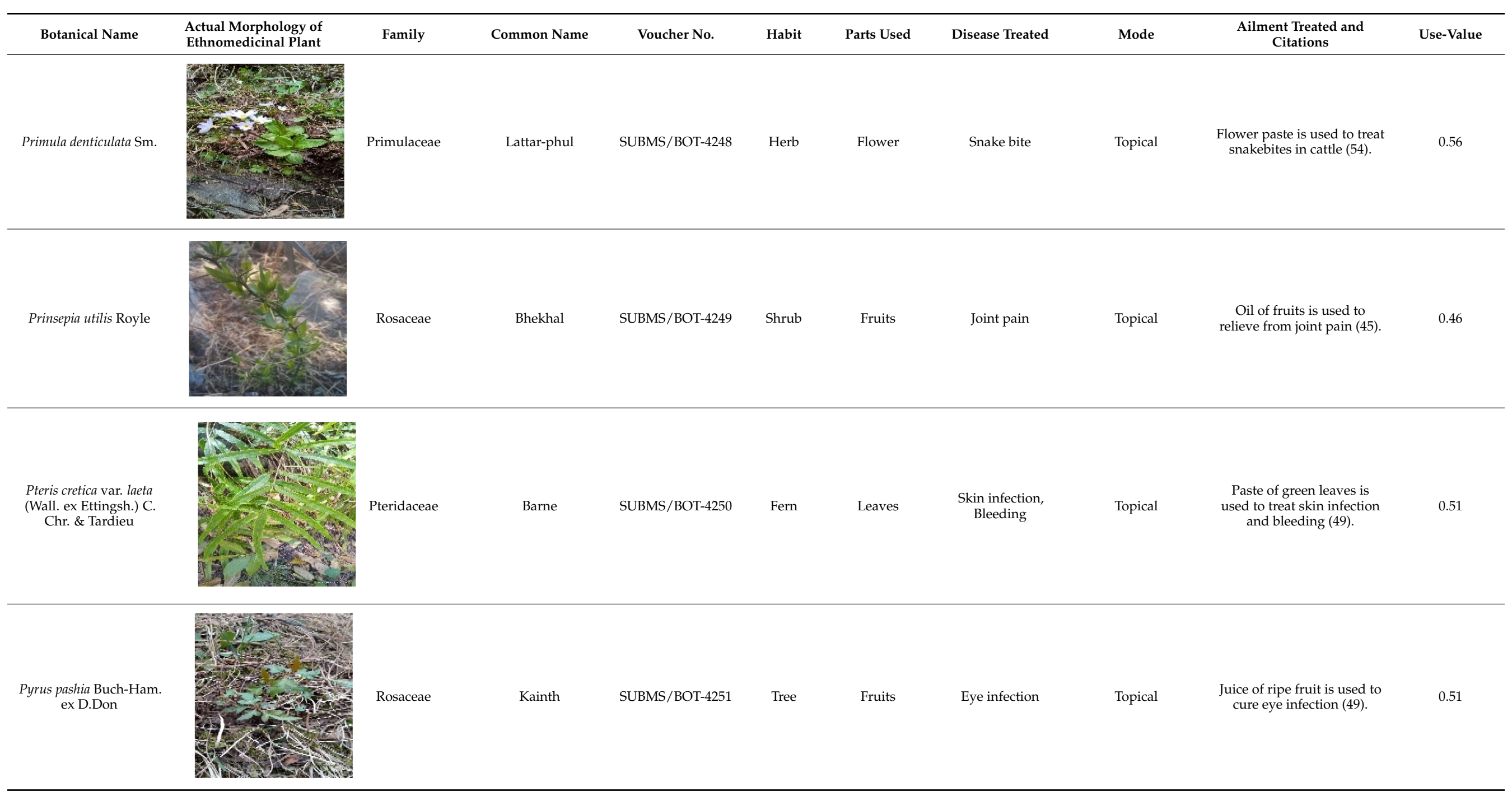


Table 2. Cont.

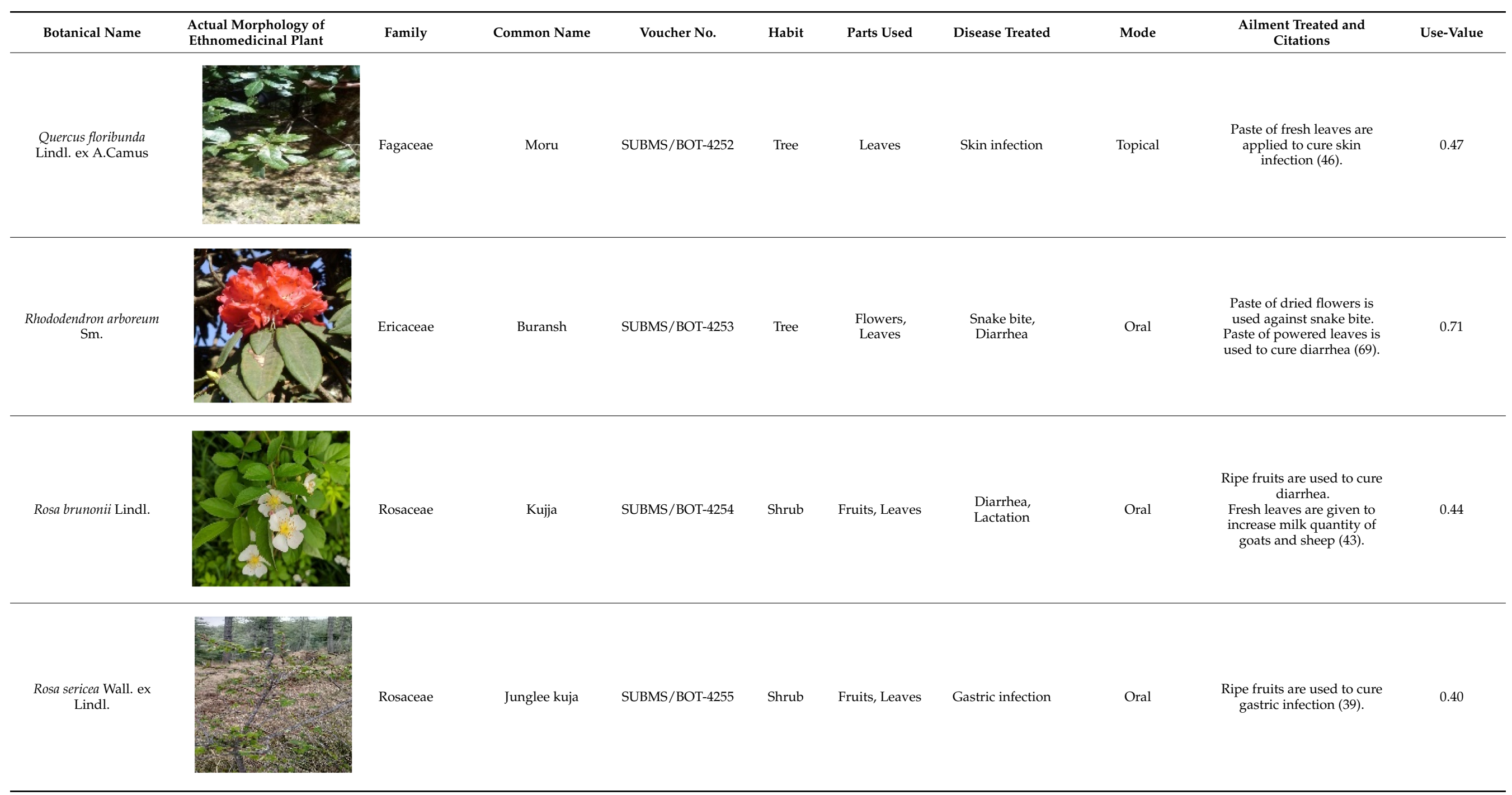


Table 2. Cont

\begin{tabular}{|c|c|c|c|c|c|c|c|c|c|c|}
\hline Botanical Name & $\begin{array}{l}\text { Actual Morphology of } \\
\text { Ethnomedicinal Plant }\end{array}$ & Family & Common Name & Voucher No. & Habit & Parts Used & Disease Treated & Mode & $\begin{array}{l}\text { Ailment Treated and } \\
\text { Citations }\end{array}$ & Use-Value \\
\hline Rubia cordifolia $\mathrm{L}$. & & Rubiaceae & Majith & SUBMS/BOT-4256 & Shrub & $\begin{array}{l}\text { Stem, } \\
\text { Leaves }\end{array}$ & $\begin{array}{l}\text { Hoof diseases, } \\
\text { Blisters }\end{array}$ & Topical & $\begin{array}{l}\text { Paste of dried root is used } \\
\text { to treat hoof diseases. } \\
\text { Paste of fresh leaves is } \\
\text { applied on blisters (44). }\end{array}$ & 0.45 \\
\hline Rubus ellipticus Sm. & & Rosaceae & Hinser & SUBMS/BOT-4257 & Shrub & Bark, Roots & $\begin{array}{l}\text { Urine infection, } \\
\text { Gastric troubles }\end{array}$ & Oral & $\begin{array}{l}\text { Juice of the root is used in } \\
\text { the diarrhea. } \\
\text { The bark from this plant is } \\
\text { used to cure urinary } \\
\text { infection (41). }\end{array}$ & 0.42 \\
\hline Rubus niveus Thunb. & & Rosaceae & Kamrai & SUBMS/BOT-4258 & Shrub & Fruits & Snake bite, Tonic & Oral & $\begin{array}{l}\text { Extracts and juices from the } \\
\text { fruits is used as an antidote } \\
\text { for snake bites and as a } \\
\text { tonic during pregnancy } \\
\text { (37). }\end{array}$ & 0.38 \\
\hline Rumex obtusifolius L. & & Polygonaceae & Kransh & SUBMS/BOT-4259 & Herb & $\begin{array}{l}\text { Leaves, } \\
\text { Roots }\end{array}$ & $\begin{array}{l}\text { Skin infection, } \\
\text { Sores }\end{array}$ & Topical & $\begin{array}{l}\text { Paste of fresh leaves is } \\
\text { applied on skin infections. } \\
\text { Paste of dried roots is } \\
\text { applied on sores (40). }\end{array}$ & 0.41 \\
\hline
\end{tabular}


Table 2. Cont.

\begin{tabular}{|c|c|c|c|c|c|c|c|c|c|c|}
\hline Botanical Name & $\begin{array}{l}\text { Actual Morphology of } \\
\text { Ethnomedicinal Plant }\end{array}$ & Family & Common Name & Voucher No. & Habit & Parts Used & Disease Treated & Mode & $\begin{array}{c}\text { Ailment Treated and } \\
\text { Citations }\end{array}$ & Use-Value \\
\hline Rumex tuberosus $\mathrm{L}$. & & Polygonaceae & Khatti patti & SUBMS/BOT-4260 & Herb & Leaves & Skin infection & Topical & $\begin{array}{l}\text { Paste of fresh leaves is } \\
\text { applied on skin infections } \\
\text { (37). }\end{array}$ & 0.38 \\
\hline Rumex hastatus D. Don & & Polygonaceae & Bhanora & SUBMS/BOT-4261 & Herb & $\begin{array}{l}\text { Leaves, } \\
\text { Tuber }\end{array}$ & Wound, Dysentery & Topical, Oral & $\begin{array}{l}\text { The extract of leaves of } \\
\text { plant is applied on wounds } \\
\text { and cuts to check bleeding. } \\
\text { The juice of the plant is } \\
\text { used in the treatment of } \\
\text { dysentery (33). }\end{array}$ & 0.34 \\
\hline Salix triandra L. & & Salicaceae & Bhaill & SUBMS/BOT-4262 & Shrub & Bark, Leaves & Fever, Joint pains & Oral, Topical & $\begin{array}{c}\text { Powder of leaves is given to } \\
\text { cure fever. } \\
\text { Paste of bark and leaves is } \\
\text { affective against joint pains } \\
\text { (36). }\end{array}$ & 0.37 \\
\hline Salvia lanata Roxb & 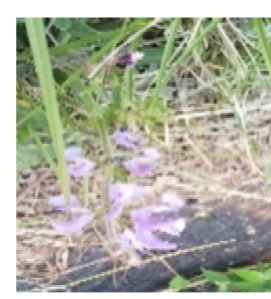 & Lamiaceae & Kuku-ro-bath & SUBMS/BOT-4263 & Herb & Leaves & Healing wounds & Topical & $\begin{array}{l}\text { Paste of fresh leaves are } \\
\text { applied on external wounds } \\
\text { (43). }\end{array}$ & 0.44 \\
\hline
\end{tabular}


Table 2. Cont

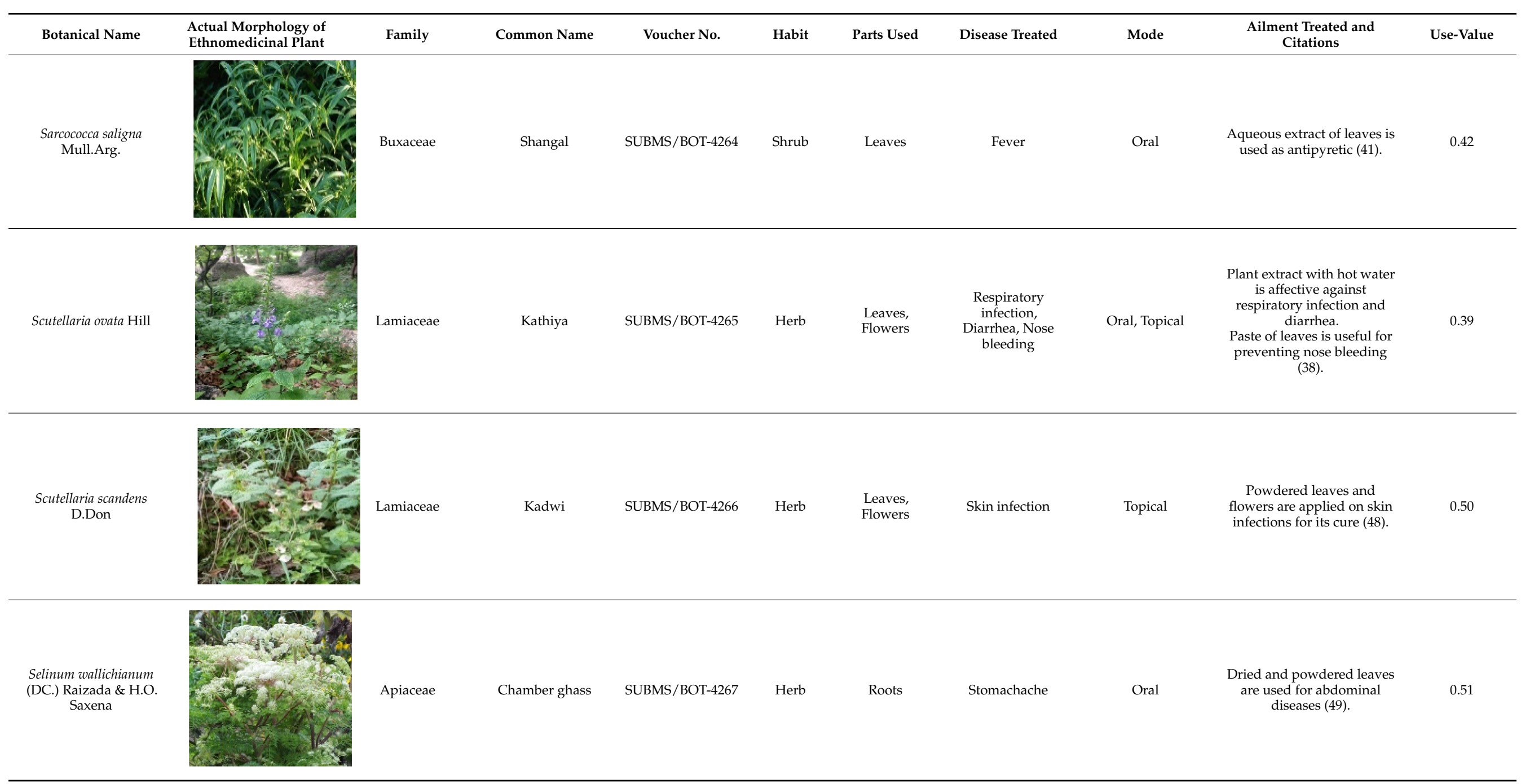


Table 2. Cont

\begin{tabular}{|c|c|c|c|c|c|c|c|c|c|c|}
\hline Botanical Name & $\begin{array}{l}\text { Actual Morphology of } \\
\text { Ethnomedicinal Plant }\end{array}$ & Family & Common Name & Voucher No. & Habit & Parts Used & Disease Treated & Mode & $\begin{array}{c}\text { Ailment Treated and } \\
\text { Citations }\end{array}$ & Use-Value \\
\hline $\begin{array}{l}\text { Silene vulgaris } \\
\text { (Moench) Garcke }\end{array}$ & & Caryophyllaceae & Monch & SUBMS/BOT-4268 & Herb & Leaves, Roots & $\begin{array}{c}\text { Vomiting, } \\
\text { Poisoning, } \\
\text { Constipation, Skin } \\
\text { infections }\end{array}$ & Topical, Oral & $\begin{array}{l}\text { Decoction of roots is used to } \\
\text { treat vomiting, poisoning } \\
\text { and constipation. } \\
\text { Liquid extract of leaves is } \\
\text { used to cure skin infections } \\
\text { (42). }\end{array}$ & 0.43 \\
\hline Solanum nigrum $\mathrm{L}$. & & Solanaceae & Genhi & SUBMS/BOT-4269 & Herb & $\begin{array}{l}\text { Leaves, } \\
\text { Flowers, } \\
\text { Roots, Fruits }\end{array}$ & $\begin{array}{l}\text { Itching, Oral ulcer, } \\
\text { Cough, Urine } \\
\text { infection }\end{array}$ & Topical, Oral & $\begin{array}{c}\text { Paste of powdered leaves, } \\
\text { fruit, flower is used against } \\
\text { itching, oral ulcers. } \\
\text { Extracts of roots and } \\
\text { flowers are used for curing } \\
\text { cough. } \\
\text { Decoction obtained from } \\
\text { leaves cure urinary troubles } \\
\text { (44). }\end{array}$ & 0.45 \\
\hline Sonchus asper (L.) Hill & & Asteraceae & Dudhiya & SUBMS/BOT-4270 & Herb & $\begin{array}{l}\text { Whole plant, } \\
\text { Leaves, Stem }\end{array}$ & Skin infection & Topical & $\begin{array}{l}\text { Freshly prepared plant } \\
\text { extract is applied on skin } \\
\text { infections (57). }\end{array}$ & 0.59 \\
\hline Sonchus brachyotus DC. & & Asteraceae & Sadhi & SUBMS/BOT-4271 & Herb & $\begin{array}{l}\text { Bud, Flowers, } \\
\text { Leaves, Stem, } \\
\text { Roots }\end{array}$ & $\begin{array}{l}\text { Puss in ear, } \\
\text { Dermatitis, } \\
\text { Ulcers }\end{array}$ & Topical, Oral & $\begin{array}{l}\text { Extract of bud is used } \\
\text { against puss formed in the } \\
\text { ear. } \\
\text { A decoction of the whole } \\
\text { plant is used to treat } \\
\text { stomach infection, itching, } \\
\text { ulcers (36). }\end{array}$ & 0.37 \\
\hline
\end{tabular}


Table 2. Cont

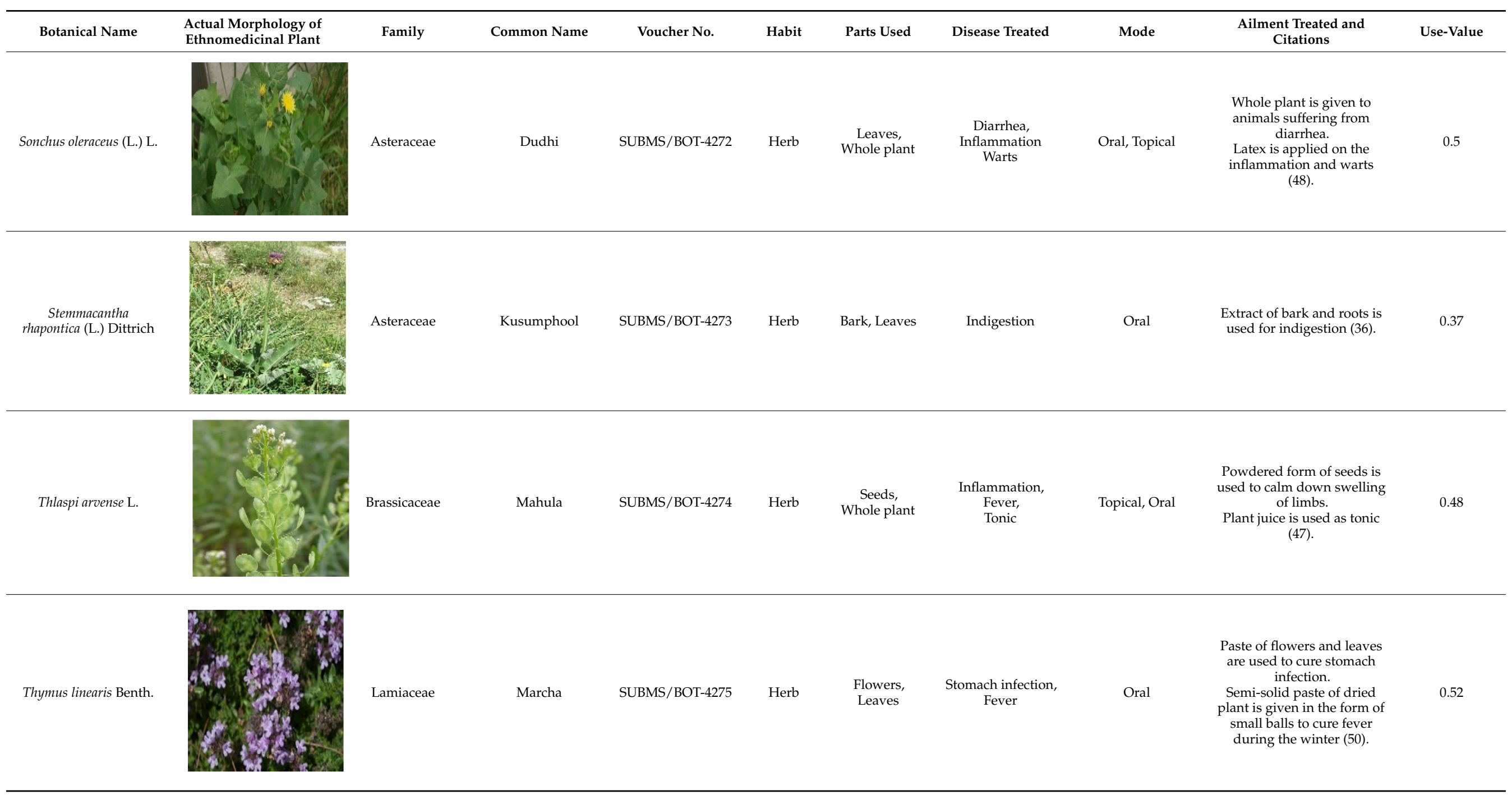


Table 2. Cont

\begin{tabular}{|c|c|c|c|c|c|c|c|c|c|c|}
\hline Botanical Name & $\begin{array}{l}\text { Actual Morphology of } \\
\text { Ethnomedicinal Plant }\end{array}$ & Family & Common Name & Voucher No. & Habit & Parts Used & Disease Treated & Mode & $\begin{array}{c}\text { Ailment Treated and } \\
\text { Citations }\end{array}$ & Use-Value \\
\hline Trifolium repens $\mathrm{L}$. & & Fabaceae & Khatti shash & SUBMS/BOT-4276 & Herb & $\begin{array}{l}\text { Leaves, } \\
\text { Flowers }\end{array}$ & $\begin{array}{l}\text { Skin infection, } \\
\text { Cough }\end{array}$ & Topical, Oral & $\begin{array}{c}\text { Paste of fresh leaves used to } \\
\text { treat skin infection. } \\
\text { Powdered flowers along } \\
\text { with leaves are used to cure } \\
\text { cough (41). }\end{array}$ & 0.42 \\
\hline Urtica dioica $\mathrm{L}$. & & Urticaceae & Kunkshi & SUBMS/BOT-4277 & Herb & Leaves & $\begin{array}{c}\text { Diarrhea, } \\
\text { Skin infection }\end{array}$ & Oral & $\begin{array}{l}\text { Semifluid paste of leaves } \\
\text { with hot water is beneficial } \\
\text { against diarrhea. } \\
\text { Leaves with other food is } \\
\text { given to cattle to get relief } \\
\text { in skin diseases during } \\
\text { lactation (39). }\end{array}$ & 0.40 \\
\hline $\begin{array}{l}\text { Valeriana jatamansi } \\
\text { Jones }\end{array}$ & & Caprifoliaceae & Mushki & SUBMS/BOT-4278 & Herb & $\begin{array}{l}\text { Leaves, } \\
\text { Rhizome }\end{array}$ & $\begin{array}{l}\text { Skin infection, } \\
\text { Wound healing, } \\
\text { Redness of eyes }\end{array}$ & Topical & $\begin{array}{l}\text { Powdered leaves are used } \\
\text { to cure skin infections. } \\
\text { Paste of root is applied on } \\
\text { wounds for better healing. } \\
\text { Rhizomes are used to treat } \\
\text { dryness and redness of eyes } \\
\text { in the cattle (52). }\end{array}$ & 0.54 \\
\hline Verbascum thapsus $\mathrm{L}$. & & Scrophulariaceae & Kukurdara & SUBMS/BOT-4279 & Herb & $\begin{array}{l}\text { Leaves, } \\
\text { Flowers, } \\
\text { Roots }\end{array}$ & $\begin{array}{l}\text { Pain, Warts, } \\
\text { Flatulence }\end{array}$ & Topical, Oral & $\begin{array}{l}\text { Decoction leaves is used to } \\
\text { treat warts on the skin. } \\
\text { Decoction of inflorescence } \\
\text { is used to cure flatulence in } \\
\text { cattle (42). }\end{array}$ & 0.43 \\
\hline
\end{tabular}


Table 2. Cont

\begin{tabular}{|c|c|c|c|c|c|c|c|c|c|c|}
\hline Botanical Name & $\begin{array}{l}\text { Actual Morphology of } \\
\text { Ethnomedicinal Plant }\end{array}$ & Family & Common Name & Voucher No. & Habit & Parts Used & Disease Treated & Mode & $\begin{array}{l}\text { Ailment Treated and } \\
\text { Citations }\end{array}$ & Use-Value \\
\hline Veronica persica Poir. & & Plantaginaceae & Raat ki kali & SUBMS/BOT-4280 & Herb & Leaves & $\begin{array}{l}\text { Skin infection, } \\
\text { Wound healing }\end{array}$ & Topical & $\begin{array}{l}\text { Paste of fresh leaves used to } \\
\text { cure skin disorders and } \\
\text { serve as an excellent wound } \\
\text { healing remedy (47). }\end{array}$ & 0.48 \\
\hline $\begin{array}{l}\text { Viburnum grandiflorum } \\
\text { Wall. ex DC. }\end{array}$ & & Adoxaceae & Pothi & SUBMS/BOT-4281 & Shrub & Leaves & Constipation & Oral & $\begin{array}{l}\text { A paste of leaves with hot } \\
\text { water is affective against } \\
\text { constipation (44). }\end{array}$ & 0.45 \\
\hline Vicia sativa $\mathrm{L}$. & & Fabaceae & Matari & SUBMS/BOT-4282 & Climber & Seeds, Leaves & Skin infection & Topical & $\begin{array}{l}\text { Paste of dry seeds and } \\
\text { leaves are used against skin } \\
\text { parasites (49). }\end{array}$ & 0.51 \\
\hline Viola canescens Wall. & & Violaceae & Banaksha & SUBMS/BOT-4283 & Herb & $\begin{array}{l}\text { Leaves, } \\
\text { Flowers, } \\
\text { Stem, Roots }\end{array}$ & $\begin{array}{l}\text { Dysentery, Cold, } \\
\text { Cough, } \\
\text { Skin infection }\end{array}$ & Oral, Topical & $\begin{array}{l}\text { Powder of whole plant with } \\
\text { hot water is used for } \\
\text { dysentery. } \\
\text { Decoction of flowers along } \\
\text { with fennel is used to cure } \\
\text { cold and cough. } \\
\text { Paste of fresh leaves and } \\
\text { stem is used to treat skin } \\
\text { infections (61). }\end{array}$ & 0.63 \\
\hline
\end{tabular}




\section{Discussion}

\subsection{Ethnoveterinary Prospective of Wild Plants}

Ethnopharmacological studies are an important step in the development of naturalsource drugs. Today, around $65 \%$ of Indian people are dependent on the medicinal plants [38]. Traditional knowledge of medicinal plant use, wild crafting and preservation promotes research for novel drugs, as well as the time effectiveness [39-42]. Medicinal plants have the capability to treat both infectious and noninfectious diseases and not only used to treat human diseases, but are also frequently utilized to treat animal ailments [43-46]. The primary goal of the ethnoveterinary study is to compile a list of plant species that have ethnoveterinary applications in the unexplored region of tehsil Chopal in district Shimla, Himachal Pradesh, India. In earlier studies, all over the world, it was documented that those medicinal plants show significant role in the human being healthcare system, as well as that of animals. They are easily available from nature, without any cost [47-49]. The documentation of ethnobotanical studies from rural and unexplored areas of all over the world is highly significant for future researchers [49-51]. In ethnoveterinary practices, plants are used for the treatment of diseases due to reduced number of side effects $[52,53]$. Ethnoveterinary medicines are easily available from surroundings without much effort [2,22]. In this study, it was found that Rosaceae family is the most frequently mentioned species from Maraog region in Shimla district. The Rosaceae plants have traditionally used to treat skin diseases, intestinal disorders, hoof infections and eye infections. In relation to shrubs and trees, herbaceous plants were used most frequently in the Maraog region. In various studies, all over the world, it was reported that different medicinal plants possess different types of phytochemicals. Phytochemical components present in different plant species are the most significant method for identifying the active medicinal potential of plants [54-57]. The concentration of phytochemicals in plant species varies according to geographical variations [58-60]. In ethnobotanical studies, it was reported that aged people have great traditional knowledge as compared to younger generation due to western culture followed by new generation $[21,61]$. The study, conducted in different regions of India, proved that new generation is not interested in traditional knowledge due to socioeconomic and cultural changes in the society [62]. The use value is a quantitative method that determines the relative importance of plant species for societies [63]. The most commonly used species had a high use value, showing that they are significant. The species with high use values found in this study had previously been scientifically well-known for their phytochemical composition and medicinal value. Saponins, tannins, flavonoids and phenolic compounds are among the chemical compounds contained in Chenopodium album, which are responsible for its antimicrobial activity [64]. The Cannabinoids, which have antiinflammatory properties, are contained in the chemical components of Cannabis sativa [65]. The phytochemical study of Cynodon dactylon revealed details of flavonoids, alkaloids, glycosides, tannins, saponins, volatile oils and flavonoids, which are responsible for its dermatological and anti-inflammatory action [66]. Similarly phenolic acids, flavonoids (quercetin, rutin) and alkaloids like berberine, berbamine, palmatine, columbamine, jatrorrhizine, oxyacanthine in Berberis aristata, Alkaloids like berberine, berbamine, chenabine, karakoramine, palmatine, baluchistanamine, gilgitine, jhelumine, punjabine, sindamine in Berberis lycium [67], pentadecanoic acid, hexadecanoic acid, heptadecanoic acid, octadecanoic acid, eicosanoic acid, steroids, amino acids (glycine, histidine) and flavonoids (tricin, kaempferol, quercetin) in Capsella bursa-pastoris [68], alkaloids (atropine, hyoscyamine, scopolamine), glycosides, saponins and tannins in Datura stramonium [69], flavonoids, terpenoids, alkaloids, carbohydrates, proteins, amino acids, steroids (phytosterols), saponins and tannins in Equisetum arvense [70], glyxylic acid, oxalic acid, vitexin, isovitexin, netural lipids, glycolipids, vitamin c, phaspholipids, fatty acids and tocopherols in Oxalis corniculate [67]. Nepalin, nepodin and rumicin in Rumex hastatus, flavonoids (catechin, epicatechin, rutin), phenolic acids (caffeic acid, gallic acid, protocatechuic acid), fatty acids (linoleic acid 67.9\%), carbohydrates (polysaccharides) in Solanum nigrum [70], taraxacin, taraxacerine, cerylalcohol, lactuce-roltaraxacin, choline, inulin, tannin, etereal oil, vitamin 
C, xanthophylls, potassium and vitamin A in Taraxacum officinale [67], alkaloids (betaine, choline), amino acids, carbohydrates, protein polymer (neutral and acidic), carotenoids (carotenes) and saponins in Urtica dioica [71]. Curculigenin in Curculigo orchioides [72], caryophyllene oxide, $\beta$-Caryophyllene, germacrene, $\beta$-Pinene in Juglans regia [55]. All of these compounds show antibacterial, antidiabetic, wound healing, hepatoprotective and anti-inflammatory activities. The majority of medicinal preparations use only one plant species, but certain medications are made with two or more plants, which improve their therapeutic effectiveness [73,74]. In the current findings, it was observed that the most frequently used plant parts were leaves followed by flowers, roots and fruits, etc. Due to harsh environmental conditions and non-availability of veterinary facilities at higher altitudes, the rural people of high altitude in Himachal Pradesh used traditional remedies to treat their livestock's illnesses [21,50].

\subsection{Prospects of Using Wild Plant Species in Horticulture}

The inhabitants were using the different plant species not only to feed and treat the diseases of their livestock, but also to nurture themselves. Plant species like Rubus niveus, Rubus ellipticus, Pyrus pashia, Prinsepia utilis, Berberis lycium, Elaeagnus umbellata and Juglans regia are well known for their fruit yields [18,75-79]. J. regia and P. pashia are important fruiting plants as they are employed by inhabitants for their fruit production, which is a good source of their income $[19,80]$. The oil extracts of $P$. utilis are consumed by locals and the remaining part of the fruit is used for feeding the cattle along with other dietary products [81-83]. It was also observed that some plant species and their products were used by locals for different agricultural and horticultural activities. The organic manure was prepared by using plants like Amaranthus blitum, Artemisia vestita, Bromus hordeaceus, Cannabis sativa, Capsella bursa-pastoris, Erigeron bonariensis, Fagopyrum acutatum, Sonchus asper and Urtica dioica along with cattle dung. The use of cannabis manure has been reported to be effective in removing harmful soil elements [84]. The plant residues were also used in the preparation of vermiwash, which is used in various agricultural and horticultural activities [85]. The paste of roots of Cirsium arvense and Rubus ellipticus was practiced to remove the root borer from the apple plant in the study region. Many researchers have reported on Rubus ellipticus for its antimicrobial activities and the extraction of silver nanoparticles which act as pathogen destroyers and root enhancers [86]. Inhabitants also showed that mulching their plant nursery with branches of Cedrus deodara and Abies pindrow was also beneficial in retaining moisture in the soil, keeping pests away and controlling weeds. Some plants were difficult to find in the wild and some was used to enhance their economy therefore, people have started cultivation of such species in their fields. For instance, P. pashia is used for the establishment of pear orchards and this species is abundant in the wild and locals collect their seeds and grow them in their fields. Similarly, it was difficult to collect herbs such as Valeriana jatamansi and Bergenia ciliata, so they are grown by the villagers in their gardens. These approaches to preserving wild plants have been employed by many communities throughout the world [87-90].

Therefore, proper documentation, conservation and pharmaceutical studies are needed to find some important medicinal plants from unexplored regions of Himachal Pradesh in India. In the literature study, it was found that Aruncus dioicus, Asplenium dalhousiae, Galinsoga quadriradiata, Gentiana argentea, Malva pusilla, Silene vulgaris and Viburnum grandiflorum were documented for the first time for ethnoveterinary purposes from the study area of Maraog region in Shimla district, Himachal Pradesh, India. Future pharmacological studies for the illnesses suggested by current survey respondents could be particularly interesting for these species. Increased interest in the chemical profile and biological activity of these species is expected as a result of this new knowledge. Their significance lies in the possibilities of discovering new medical plant uses and herbal therapies in veterinary medicine. 


\section{Conclusions}

The rural people of Maraog region in tehsil Chopal of district Shimla in Himachal Pradesh, India, used ethnoveterinary plants to treat their livestock diseases. The current study documents the ethnoveterinary medicines, which can be used as a database for scientific research studies in the future. It was observed that, from all categories of plants, herbs and shrubs were the most documented ethnoveterinary plants by the native informants of study site. In this study, most of the plants are first time documented for ethnoveterinary purposes from Maraog region in tehsil Chopal of district Shimla, so its urgent need to document different medicinal plants from unexplored regions of Himachal Pradesh. In the study area, internally and externally medicinal formulations were used to treat livestock illnesses. The male informants of the study site possess good traditional knowledge as compared to the female informants. The rural people reported that new generation is not much interested in traditional knowledge due to the social, cultural and modernization, so it is urgently needed to document and conserve ethnomedicinal plants used for both human and livestock diseases in the rural areas of Shimla district, Himachal Pradesh, India.

Supplementary Materials: The following are available online at https: / www.mdpi.com/article/ 10.3390/horticulturae7100351/s1, Supplementary Information S1: (A) Demographic Data, (B) Ethnoveterinary Plant Uses and (C) Informants Declaration.

Author Contributions: Conceptualization, Resources, Supervision, writing —original draft preparation: M.K., S.D., R., S.S. and S.P. (Sunil Puri); Methodology, Investigation and writing—original draft preparation: P.P., R., A.P., R.J., M.A. (Mansoor Ali), M.T., N.K., S.P. (Suraj Prakash), S.R. (Sonia Rathour) and S.J.; Software, writing-review and editing and Formal Analysis: S.P.B., D.C., C.S., S.R. (Sureshkumar Rajalingam), M.S., S.D., S.S., S.C., S.N., M.K.S., R.D.D., M.A. (M. Alblihed), A.F.E.-k. and M.M.A.-D.; Visualization: S.P.B., M.K. and M.S. All authors have read and agreed to the published version of the manuscript.

Funding: This research received no external funding.

Institutional Review Board Statement: Not applicable.

Informed Consent Statement: Not applicable.

Data Availability Statement: Data is contained within the article or Supplementary Materials.

Acknowledgments: The authors are highly thankful to BSI, Dehradun for identification of plant samples. This work was supported by the Taif University Researchers Supporting Program (Project number: TURSP-2020/93), Taif University, Saudi Arabia. Also, the authors would like to thank the Deanship of Scientific Research at King Khalid University, Abha, KSA for funding this work under Grant number (R.G.P.2/47/42).

Conflicts of Interest: The authors confirm no conflict of interest.

\section{References}

1. Barboza, R.R.; De MS Souto, W.; Da S Mourão, J. The use of zootherapeutics in folk veterinary medicine in the district of Cubati, Paraíba State, Brazil. J. Ethnobiol. Ethnomed. 2007, 3, 1-14. [CrossRef]

2. McGaw, L.J.; Famuyide, I.M.; Khunoana, E.T.; Aremu, A.O. Ethnoveterinary botanical medicine in South Africa: A review of research from the last decade (2009 to 2019). J. Ethnopharmacol. 2020, 257, 112864. [CrossRef]

3. Greene, A.M.; Panyadee, P.; Inta, A.; Huffman, M.A. Asian elephant self-medication as a source of ethnoveterinary knowledge among Karen mahouts in northern Thailand. J. Ethnopharmacol. 2020, 259, 112823. [CrossRef]

4. Oliveira, M.; Hoste, H.; Custódio, L. A systematic review on the ethnoveterinary uses of mediterranean salt-tolerant plants: Exploring its potential use as fodder, nutraceuticals or phytotherapeutics in ruminant production. J. Ethnopharmacol. 2020, 267, 113464. [CrossRef]

5. World Health Organization. World Health Organization General Guidelines for Methodologies on Research and Evaluation of Traditional Medicine; WHO: Geneva, Switzerland, 2000.

6. Dhiman, N.; Shivani, Y.S.T.; Kumar, S. Diversity of ethnomedicinal plants in Churdhar Wildlife Sanctuary of district Sirmour of Himachal Pradesh, India. J. Appl. Pharm. Sci. 2019, 9, 48-53.

7. Tangjang, S.; Namsa, N.D.; Aran, C.; Litin, A. An ethnobotanical survey of medicinal plants in the Eastern Himalayan zone of Arunachal Pradesh, India. J. Ethnopharmacol. 2011, 134, 18-25. [CrossRef] [PubMed] 
8. Saunders, R.M. Monograph of Schisandra (Schisandraceae); American Society of Plant Taxonomists: Ann Arbor, MI, USA, 2000.

9. Sharma, V. Diversity of Plant and Soil Nematodes in Uttarakhand, India. In Pests of Forest Importance E Their Management; Tyagi, B.K., Veer, V., Prakash, S., Eds.; Scientific Publishers: Jodhpur, India, 2008; p. 251.

10. Mekhemar, M.; Geib, M.; Kumar, M.; Radha, S.P.; Hassan, Y.; Dörfer, C. Salvadora persica: Nature's Gift for Periodontal Health. Antioxidants 2021, 10, 712. [CrossRef] [PubMed]

11. Kumar, M.; Changan, S.; Tomar, M.; Prajapati, U.; Saurabh, V.; Hasan, M.; Sasi, M.; Maheshwari, C.; Singh, S.; Dhumal, S.; et al . Custard apple (Annona squamosal L.) leaves: Nutritional composition, phytochemical profile, and health-promoting biological activities. Biomolecules 2021, 11, 614. [CrossRef] [PubMed]

12. Wani, Z.A.; Kumar, N. Akash Ethnobotanical Study of Some Threatened Plants in District Baramulla, Kashmir, Jammu and Kashmir, India. Int. J. Curr. Res. Biosci. Plant Biol. 2016, 3, 58-64. [CrossRef]

13. Pandey, D.K.; Dey, A. A validated and densitometric HPTLC method for the simultaneous quantification of reserpine and ajmalicine in Rauvolfia serpentina and Rauvolfia tetraphylla. Rev. Bras. Farm. 2016, 26, 553-557. [CrossRef]

14. Radha, S.P.; Puri, S. Assessment of wild medicinal plant used by migratory shepherds in alpine area of Rakchham-Chitkul Wildlife Sanctuary of district Kinnaur in Himachal Pradesh. Plant Arch. 2019, 19, 418-429.

15. Kumar, M.; Tomar, M.; Punia, S.; Grasso, S.; Arrutia, F.; Choudhary, J.; Singh, S.; Verma, P.; Mahapatra, A.; Patil, S.; et al. Cottonseed: A sustainable contributor to global protein requirements. Trends Food Sci. Technol. 2021, 111, 100-113. [CrossRef]

16. Radha, S.P.; Chauhan, P.; Puri, S.; Sharma, A.K.; Pundir, A. A study of wild medicinal plants used in Nargu Wildlife Sanctuary of district Mandi in Himachal Pradesh, India. J. Appl. Pharm. Sci. 2021, 11, 135-144.

17. Singh, R.P.; Prakash, S.; Bhatia, R.; Negi, M.; Singh, J.; Bishnoi, M.; Kondepudi, K.K. Generation of structurally diverse pectin oligosaccharides having prebiotic attributes. Food Hydrocoll. 2020, 108, 105988. [CrossRef]

18. Maikhuri, R.K.; Semwal, R.L.; Singh, A.; Nautiyal, M.C. Wild fruits as a contribution to sustainable rural development: A case study from the Garhwal Himalaya. Int. J. Sustain. Dev. World Ecol. 1994, 1, 56-68. [CrossRef]

19. Singh, V.; Dasgupta, S.; Jhaldiyal, V.; Chauhan, D.; Todaria, N. Diversity pattern of vegetation in and around proposed Kotlibhel hydroelectric project along the Alaknanda River in Garhwal Himalaya (India). iForest Biogeosci. For. 2011, 4, 38-43. [CrossRef]

20. Monteiro, M.V.; Bevilaqua, C.M.; Palha, M.D.; Braga, R.R.; Schwanke, K.; Rodrigues, S.T.; Lameira, O.A. Ethnoveterinary knowledge of the inhabitants of Marajó Island, Eastern Amazonia, Brazil. Acta Amaz. 2011, 41, 233-242. [CrossRef]

21. Radha, S.P.; Pundir, A. Survey of wild medicinal plants used by migratory shepherds in Summer Hill of District Shimla in Himachal Pradesh. Bio Bull. 2019, 5, 18-24.

22. Mathias, E. Ethnoveterinary medicine in the era of evidence-based medicine: Mumbo-jumbo, or a valuable resource? Vet. J. 2006, 173, 241-242. [CrossRef] [PubMed]

23. Ritter, R.A.; Monteiro, M.V.B.; Monteiro, F.; Rodrigues, S.T.; Soares, M.L.; Silva, J.C.R.; Palha, M.D.D.C.; Biondi, G.F.; Rahal, S.; Tourinho, M.M. Ethnoveterinary knowledge and practices at Colares island, Pará state, eastern Amazon, Brazil. J. Ethnopharmacol. 2012, 144, 346-352. [CrossRef]

24. Khattak, N.S.; Nouroz, F.; Rahman, I.U.; Noreen, S. Ethnoveterinary uses of medicinal plants of district Karak, Pakistan. J. Ethnopharmacol. 2015, 171, 273-279. [CrossRef]

25. González, J.A.; Vallejo, J.R. The use of domestic animals and their derivative products in contemporary Spanish ethnoveterinary medicine. J. Ethnopharmacol. 2021, 271, 113900. [CrossRef]

26. Bhandari, P.R.; Mukerji, B. Role of indigenous drugs in veterinary medicine in India. Indian Vet. J. 1958, 1, 55.

27. Mazars, G. Traditional veterinary medicine in India. Rev. Sci. Tech. 1994, 13, 443-451.

28. Assefa, A.; Bahiru, A. Ethnoveterinary botanical survey of medicinal plants in Abergelle, Sekota and Lalibela districts of Amhara region, Northern Ethiopia. J. Ethnopharmacol. 2018, 213, 340-349. [CrossRef] [PubMed]

29. Singh, J.; Singh, J.; Sharma, D. Traditional wisdom to treat the most common ailments in Chopal region of Shimla district, Himachal Pradesh, India. Plant Arch. 2018, 18, 2759-2769.

30. Jain, S.K. Ethnobotany. Interdiscip. Sci. Rev. 1986, 11, 285-292. [CrossRef]

31. Namsa, N.D.; Tag, H.; Mandal, M.; Kalita, P.; Das, A. An ethnobotanical study of traditional anti-inflammatory plants used by the Lohit community of Arunachal Pradesh, India. J. Ethnopharmacol. 2009, 125, 234-245. [CrossRef]

32. Phillips, O.; Gentry, A.H. The useful plants of Tambopata, Peru: I. Statistical hypotheses tests with a new quantitative technique. Econ. Bot. 1993, 47, 15-32. [CrossRef]

33. Rossato, S.C.; Leitao-Filho, H.; Gegossi, A. Ethnobotany of Caicaras of the Atlantic Forest coast (Brazil). Econ. Bot. 1999, 53, 387-395. [CrossRef]

34. Albuquerque, U.P.; Lucena, R.F.; Monteiro, J.M.; Florentino, A.T.; Cecília de Fátima, C.B.R. Evaluating Two Quantitative Ethnobotanical Techniques. Ethnobot. Res. Appl. 2006, 4, 51-60. [CrossRef]

35. Yabesh, J.M.; Prabhu, S.; Vijayakumar, S. An ethnobotanical study of medicinal plants used by traditional healers in silent valley of Kerala, India. J. Ethnopharmacol. 2014, 154, 774-789. [CrossRef] [PubMed]

36. Da Silva, V.A.; Andrade, L.D.; De Albuquerque, U.P. Revising the cultural significance index: The case of the Fulni-ô in northeastern Brazil. Field Methods 2006, 18, 98-108. [CrossRef]

37. Musa, M.S.; Abdelrasool, F.E.; Elsheikh, E.A.; Ahmed, L.A.; Mahmoud, A.L.; Yagi, S.M. Ethnobotanical study of medicinal plants in the Blue Nile State, South-eastern Sudan. J. Med. Plants Res. 2011, 5, 4287-4297. 
38. Radha, S.P.; Puri, S.; Pundir, A. Review on Ethnomedicinal Plant: Trillium govanianum Wall. Ex, D. Don. Int. J. Theor. Appl. Sci. 2019, 11, 4-9.

39. Lans, C. Possible similarities between the folk medicine historically used by First Nations and American Indians in North America and the ethnoveterinary knowledge currently used in British Columbia, Canada. J. Ethnopharmacol. 2016, 192, 53-66. [CrossRef] [PubMed]

40. Stucki, K.; Dal Cero, M.; Vogl, C.R.; Ivemeyer, S.; Meier, B.; Maeschli, A.; Hamburger, M.; Walkenhorst, M. Ethnoveterinary contemporary knowledge of farmers in pre-alpine and alpine regions of the Swiss cantons of Bern and Lucerne compared to ancient and recent literature-is there a tradition? J. Ethnopharmacol. 2019, 234, 225-244. [CrossRef]

41. Abo-EL-Sooud, K. Ethnoveterinary perspectives and promising future. Int. J. Vet. Sci. Med. 2018, 6, 1-7. [CrossRef]

42. Marković, M.S.; Pljevljakušić, D.S.; Nikolić, B.M.; Miladinović, D.L.; Djokić, M.M.; Rakonjac, L.B.; Jovanović, V.P.S. Ethnoveterinary knowledge in Pirot County (Serbia). S. Afr. J. Bot. 2021, 137, 278-289. [CrossRef]

43. González, J.A.; Amich, F.; Postigo-Mota, S.; Vallejo, J.R. The use of wild vertebrates in contemporary Spanish ethnoveterinary medicine. J. Ethnopharmacol. 2016, 191, 135-151. [CrossRef]

44. Miara, M.D.; Bendif, H.; Ouabed, A.; Rebbas, K.; Hammou, M.A.; Amirat, M.; Greene, A.; Teixidor-Toneu, I. Ethnoveterinary remedies used in the Algerian steppe: Exploring the relationship with traditional human herbal medicine. J. Ethnopharmacol. 2019, 244, 112164. [CrossRef] [PubMed]

45. Radha, S.P.; Puri, S.; Kumar, V. Phytochemical screening of medicinal plants used by tribal migratory shepherds in Western Himalaya. Ann. Biol. 2019, 35, 11-14.

46. Radha, S.P.; Kumar, M.; Puri, S.; Pundir, A.; Bangar, S.P.; Changan, S.; Choudhary, P.; Parameswari, E.; Alhariri, A.; Samota, M.K.; et al. Evaluation of nutritional, phytochemical, and mineral composition of selected medicinal plants for therapeutic uses from Cold Desert of Western Himalaya. Plants 2021, 10, 1429. [CrossRef]

47. Njoroge, G.N.; Bussmann, R.W. Herbal usage and informant consensus in ethnoveterinary management of cattle diseases among the Kikuyus (Central Kenya). J. Ethnopharmacol. 2006, 108, 332-339. [CrossRef]

48. Grade, J.T.; Tabuti, J.R.; Van Damme, P. Ethnoveterinary knowledge in pastoral Karamoja, Uganda. J. Ethnopharmacol. 2009, 122, 273-923. [CrossRef]

49. Dilshad, S.M.; Iqbal, Z.; Muhammad, G.; Iqbal, A.; Ahmed, N. An inventory of the ethnoveterinary practices for reproductive disorders in cattle and buffaloes, Sargodha district of Pakistan. J. Ethnopharmacol. 2008, 117, 393-402. [CrossRef]

50. Radha, S.P.; Pundir, A. Survey of ethnomedicinal plants used by migratory shepherds in Shimla district of Himachal Pradesh. Plant Arch. 2019, 19, 477-482.

51. Radha, S.P.; Puri, S. Study of wild medicinal plants used by tribal migratory shepherds in hills of Shimla District, Himachal Pradesh. Plant Arch. 2019, 19, 785-790.

52. Alawa, J.; Jokthan, G.; Akut, K. Ethnoveterinary medical practice for ruminants in the subhumid zone of northern Nigeria. Prev. Veter. Med. 2002, 54, 79-90. [CrossRef]

53. Tabuti, J.R.; Dhillion, S.S.; Lye, K.A. Ethnoveterinary medicines for cattle (Bos indicus) in Bulamogi county, Uganda: Plant species and mode of use. J. Ethnopharmacol. 2003, 88, 279-286. [CrossRef]

54. Radha, S.P.; Janjua, S.; Srivastava, S.; Negi, V. Ethnobotanical study of medicinal plants used in Shikari Devi Wildlife Sanctuary of Himachal Pradesh, India. Med. Plants 2020, 12, 666-673.

55. Kumar, M.; Prakash, S.; Radha, S.P.; Kumari, N.; Pundir, A.; Punia, S.; Saurabh, V.; Choudhary, P.; Changan, S.; Dhumal, S.; et al. Beneficial role of antioxidant secondary metabolites from medicinal plants in maintaining oral health. Antioxidants 2021, 10, 1061. [CrossRef]

56. Kumar, M.; Tomar, M.; Saurabh, V.; Sasi, M.; Punia, S.; Potkule, J.; Maheshwari, C.; Changan, S.; Radha, S.P.; Bhushan, B.; et al. Delineating the inherent functional descriptors and biofunctionalities of pectic polysaccharides. Carbohydr. Polym. 2021, 269, 118319. [CrossRef] [PubMed]

57. Kumar, M.; Potkule, J.; Patil, S.; Mageshwaran, V.; Radha, S.P.; Satankar, V.; Berwal, M.K.; Mahapatra, A.; Saxena, S.; Ashtaputre, N.; et al. Evaluation of detoxified cottonseed protein isolate for application as food supplement. Toxin Rev. 2021, 13, 1-8. [CrossRef]

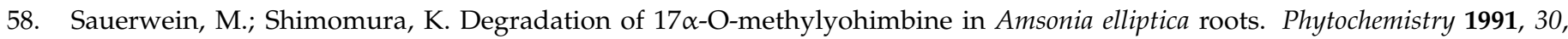
1449-1450. [CrossRef]

59. Arabhosseini, A.; Huisman, W.; Van Boxtel, A.; Müller, J. Long-term effects of drying conditions on the essential oil and color of tarragon leaves during storage. J. Food Eng. 2007, 79, 561-566. [CrossRef]

60. Karabulut, I.; Topcu, A.; Duran, A.; Turan, S.; Ozturk, B. Effect of hot air drying and sun drying on color values and $\beta$-carotene content of apricot (Prunus armenica L.). LWT Food Sci. Technol. 2007, 40, 753-758. [CrossRef]

61. Radha, S.P.; Puri, S. Phytochemical analysis of ethanolic extracts of leaves of some selected medicinal plants used by tribal community of Sangla Valley, District Kinnaur, Himachal Pradesh. Plant Arch. 2019, 19, 397-403.

62. Jagtap, S.; Deokule, S.; Bhosle, S. Some unique ethnomedicinal uses of plants used by the Korku tribe of Amravati district of Maharashtra, India. J. Ethnopharmacol. 2006, 107, 463-469. [CrossRef]

63. Vendruscolo, G.S.; Mentz, L.A. Study of the concordance of the citations of use and importance of the species and families used as medicinal by the community of the Ponta Grossa neighborhood, Porto Alegre, RS, Brazil. Acta Bot. Bras. 2006, 20, 367-382. [CrossRef] 
64. Al-Snafi, A.E. The chemical constituents and pharmacological effects of Chenopodium album-An overview. Int. J. Pharmacol. Screen. Methods 2015, 5, 10-17.

65. Shakil, S.S.; Gowan, M.; Hughes, K.; Azam, M.N.; Ahmed, M.N. A narrative review of the ethnomedicinal usage of Cannabis sativa Linnaeus as traditional phytomedicine by folk medicine practitioners of Bangladesh. J. Cannabis Res. 2021, 3, 1-2. [CrossRef]

66. Al-Snafi, A.E. Chemical constituents and pharmacological effects of Cynodon dactylon-A Review. IOSR J. Pharm. (IOSRPHR) 2016, 6, 17-31. [CrossRef]

67. Abbasi, A.M.; Khan, M.A.; Ahmad, M.; Zafar, M.; Khan, H.; Muhammad, N.; Sultana, S. Medicinal plants used for the treatment of jaundice and hepatitis based on socio-economic documentation. Afr. J. Biotechnol. 2009, 8.

68. Al-Snafi, A.E. The chemical constituents and pharmacological effects of Capsella bursa-pastoris-A review. Int. J. Pharmacol. Toxicol. 2015, 5, 76-81.

69. Al-Snafi, A.E. The pharmacology of Equisetum arvense-A review. IOSR J. Pharm. (IOSRPHR) 2017, 7, 31-42. [CrossRef]

70. Singh, K.; Lal, B. Ethnomedicines used against four common ailments by the tribal communities of Lahaul-Spiti in western Himalaya. J. Ethnopharmacol. 2008, 115, 147-159. [CrossRef]

71. Chauhan, R.; Ruby, K.M.; Shori, A.; Dwivedi, J. Solanum nigrum with dynamic therapeutic role: A review. Int. J. Pharm. Sci. Res. 2012, 15, 65-71.

72. Girish, C.; Pradhan, S. Indian herbal medicines in the treatment of liver diseases: Problems and promises. Fundam. Clin. Pharmacol. 2012, 26, 180-189. [CrossRef] [PubMed]

73. Giday, M.; Teklehaymanot, T.; Animut, A.; Mekonnen, Y. Medicinal plants of the Shinasha, Agew-awi and Amhara peoples in northwest Ethiopia. J. Ethnopharmacol. 2007, 110, 516-525. [CrossRef] [PubMed]

74. Muthu, C.; Ayyanar, M.; Raja, N.; Ignacimuthu, S. Medicinal plants used by traditional healers in Kancheepuram District of Tamil Nadu, India. J. Ethnobiol. Ethnomed. 2006, 2, 1-10. [CrossRef]

75. Nesello, L.A.; Beleza, M.L.; Mariot, M.; Mariano, L.N.; De Souza, P.; Campos, A.; Cechinel-Filho, V.; Andrade, S.F.; Da Silva, L.M. Gastroprotective value of berries: Evidences from methanolic extracts of Morus nigra and Rubus niveus fruits. Gastroenterol. Res. Pract. 2017, 2017, 7089697. [CrossRef]

76. Rahimi Madiseh, M.; Heidarian, E.; Rafieian-Kopaei, M. Biochemical components of Berberis lycium fruit and its effects on lipid profile in diabetic rats. J. HerbMed Pharmacol. 2014, 3, 15-19.

77. Chauhan, P.S.; Bisht, S.; Ahmed, S. Traditional and ethnobotanical uses of medicinal trees in district Tehri Garhwal (Western Himalayas). Int. J. Ayurvedic Herb. Med. 2017, 7, 2442-2448.

78. Singh, J.; Dhupper, R.; Sharma, S. Survey of ethnomedicinal plants used by indigenous people of Nerwa range, Chopal forest division, Himachal Pradesh, India. Med. Plants Int. J. Phytomed. Relat. Ind. 2020, 12, 381-391. [CrossRef]

79. Saklani, S.; Chandra, S.; Badoni, P.P.; Dogra, S. Antimicrobial activity, nutritional profile and phytochemical screening of wild edible fruit of Rubus ellipticus. Int. J. Med. Aromat. Plants 2012, 2, 269-274.

80. Shigaeva, J.; Darr, D. On the socio-economic importance of natural and planted walnut (Juglans regia L.) forests in the Silk Road countries: A systematic review. For. Policy Econ. 2020, 118, 102233. [CrossRef]

81. Chen, P.; Jiang, W.; Liu, Y.; Yan, F.; Chen, F. Main physical chemistry characteristics and fatty acid composition of three plant oils and their bio-diesel fuels. Guangxi Zhiwu/Guihaia 2007, 27, 448-452.

82. Peng, Z. Advances in Research of the Prinsepia utilis Royle In China. J. Anhui Agric. Sci. 2011, $2011,24$.

83. Maikhuri, R.K.; Singh, A.; Semwal, R.L. Prinsepia utilis Royle: A wild, edible oil shrub of the higher Himalayas. Plant Genet. Resour. Newslett. 1994, 98, 5-8.

84. Singani, A.A.S.; Ahmadi, P. Manure Application and Cannabis Cultivation Influence on Speciation of Lead and Cadmium by Selective Sequential Extraction. Soil Sediment Contam. Int. J. 2012, 21, 305-321. [CrossRef]

85. Gudeta, K.; Julka, J.; Kumar, A.; Bhagat, A.; Kumari, A. Vermiwash: An agent of disease and pest control in soil, a review. Heliyon 2021, 7, e06434. [CrossRef]

86. Khan, S.; Ali, A.S.; Ali, S.A. Green nanotechnology a boon in silver nanoparticle (AgNPs) synthesis-certain aspects of AgNPs biomedical applications and an outline of its toxicological impacts-A mini review. Eur. J. Pharm. Med. Res. 2020, 7, $261-273$.

87. Negi, P.S.; Subramani, S.P. Wild edible plant genetic resources for sustainable food security and livelihood of Kinnaur district, Himachal Pradesh, India. Int. J. Conserv. Sci. 2015, 6, 657-668.

88. Kumar, G.; Duggal, S. Ethnomedicinal diversity of aromatic plants in foot hill regions of Himachal Pradesh, India. Int. J. Theor. Appl. Sci. 2019, 11, 18-39.

89. Fitzpatrick, J.T. Cultivating and Preserving American Wild Flowers, 1890-1965; Cornell University: Ithaca, NY, USA, 2006.

90. Shigeta, M. Folk in-situ conservation of ensete [Ensete ventricosum (Welw.) EE Cheesman]: Toward the interpretation of indigenous agricultural science of the Ari, Sowthwestern Ethiopia. Afr. Study Monogr. 1990, 10, 93-107. 